ANL-HEP-PR-01-112

CERN-TH/2001-307

EFI-2001-052

FERMILAB-Pub-01/353-T

MC-TH-2001-011

hep-ph/0111245

November 2001

\title{
Higgs-Boson Pole Masses in the MSSM with Explicit CP Violation
}

\author{
M. Carena ${ }^{a}$, J. Ellis $^{b}$, A. Pilaftsis ${ }^{c}$ and C.E.M. Wagner ${ }^{d, e}$ \\ ${ }^{a}$ Fermilab, P.O. Box 500, Batavia IL 60510, U.S.A. \\ ${ }^{b}$ Theory Division, CERN, CH-1211 Geneva 23, Switzerland \\ ${ }^{c}$ Department of Physics and Astronomy, University of Manchester, \\ Manchester M13 9PL, United Kingdom \\ ${ }^{d}$ High Energy Physics Division, Argonne National Lab., Argonne IL 60439, U.S.A. \\ ${ }^{e}$ Enrico Fermi Institute, University of Chicago, 5640 Ellis Ave., Chicago IL 60637, U.S.A.
}

\begin{abstract}
Extending previous results obtained in the effective-potential approach, we derive analytic expressions for the pole masses of the charged and neutral Higgs bosons in the minimal supersymmetric standard model (MSSM) with explicit CP violation. In such a minimal supersymmetric model, the CP invariance of the Higgs potential is explicitly broken by quantum effects dominated by third-generation squarks at the one-loop level and by oneloop gluino- and higgsino-mediated threshold corrections to the top- and bottom-quark Yukawa couplings at the two-loop level. Field-theoretic issues arising in the description of a two-level Higgs-boson system strongly mixed due to CP violation are addressed. Numerical results for the pole masses of the $\mathrm{CP}$-violating Higgs bosons are presented, and comparisons are made with previous results obtained in the effective-potential approach.
\end{abstract}




\section{Introduction}

The minimal supersymmetric extension of the Standard Model (MSSM) represents the most economical, and perhaps the most appealing, low-energy realization of supersymmetry (SUSY) softly broken at $0.1-1 \mathrm{TeV}$ energies, within which a natural formulation of the gauge-hierarchy problem is possible [1]. Unlike the Standard Model (SM), the MSSM exhibits gauge-coupling unification at very high energy scales of order $10^{16} \mathrm{GeV}$ [2], offers a theoretical framework able to realize cosmological baryogenesis via a strongly first-order electroweak phase transition [3, [4, and provides an interesting candidate for the cold dark matter favoured by astrophysics [5]. Moreover, an important prediction of the MSSM is the existence of a light neutral Higgs boson which, in general, has SM-like properties and whose mass cannot exceed a calculable upper bound of about $130 \mathrm{GeV}$ [6]. Since supersymmetric particle contributions to the precision electroweak observables rapidly decouple for values of the sparticle masses above the weak scale, these values of the lightest neutral Higgsboson mass are consistent with the analysis of precision electroweak data, which, within the Standard Model, lead to a preference for a relatively Higgs-boson mass, not larger than $\sim 240 \mathrm{GeV}$ [7].

It has been emphasized in many papers that loop effects dominated by thirdgeneration squarks may violate sizeably the tree-level CP invariance of the MSSM Higgs

potential 18, 9, 10, 11, 12, 13, 14, 15, 16, 17, 18], giving rise to important modifications of the Higgs-boson couplings to the $W^{ \pm}$and $Z$ bosons and to fermionic matter. Radiative CP violation in the MSSM Higgs sector thereby affects significantly the production rates of the Higgs bosons. In particular, modifications of the couplings to the $Z$ boson of the lightest and second-lightest Higgs bosons might allow a relatively light Higgs boson with a mass of 60-70 GeV to have escaped detection at LEP 2 [12]. The upgraded Tevatron collider, the LHC and a linear $e^{+} e^{-}$collider will be able to probe in detail this minimal CP-violating supersymmetric model.

An accurate determination of the MSSM Higgs-boson mass spectrum in the presence of $\mathrm{CP}$ violation plays an important rôle in the studies of electroweak baryogenesis [四]. It may also be important for understanding the domain of MSSM parameter space compatible with supersymmetric dark matter [5, since $\mathrm{CP}$ violation is known to extend the allowed ranges of parameters, and even in the CP-conserving case rapid annihilation of relic particles via direct-channel Higgs poles can yield 'funnels' of parameter space extending to exceptionally large sparticle masses.

In earlier work, we computed the charged and neutral Higgs-boson masses from the 
one-loop effective MSSM Higgs potential, utilizing renormalization-group (RG) methods. Here, we extend our earlier computation of RG-improved Higgs-boson masses by taking into account finite contributions that arise from the shifts in the positions of the poles in the corresponding Higgs-boson propagators. Even though the computation of the Higgsboson pole masses that we make is diagrammatic at the one-loop level, we are still able to implement the RG techniques known from previous effective-potential analyses in the MSSM [19, 20, 21, 22]. In fact, one can establish a close relationship between the RGimproved effective-potential approach followed in [12] and the RG-improved diagrammatic calculation presented here. Specifically, if one considers the zero-momentum limit of the Higgs-boson propagators within the diagrammatic framework, the two approaches give identical results, which constitutes a non-trivial check on the correctness of our numerical evaluations.

Following [12], we include in our RG-improved diagrammatic approach the leading two-loop logarithms due to QCD corrections, as well as the leading two-loop logarithms induced by the top- and bottom-quark Yukawa couplings [22]. In addition, we take into consideration the leading one-loop logarithms due to gaugino and higgsino quantum effects 23. Finally, we add to the effective potential the potentially large two-loop non-logarithmic corrections originating from one-loop threshold effects on the top- and bottom-quark Yukawa couplings, associated with the decoupling of the third-generation squarks [24].

As we show below, sparticle loops may have a relevant impact on the definition of the pole masses. Although the shift of the lightest neutral Higgs-boson mass is typically of the order of a few $\mathrm{GeV}$, and therefore of the order of the uncertainties implicit in the effective potential computation of the neutral Higgs masses, the shift of the heavier neutral and charged Higgs-boson masses may be much larger, of the order of a few tens of GeV. These relatively large differences between the pole and running masses are usually associated with Higgs-boson mass values which are close to the thresholds for on-shell production of the third-generation squarks, and may have important consequences for Higgs physics at future colliders.

The organization of the paper is as follows. Section 2 describes the approach we use to evaluate the Higgs-boson pole masses. Based on this approach, we compute in Section 3 the pole masses of the charged and neutral Higgs bosons. Analytic expressions useful in the computation are given in Appendices $\mathrm{A}$ and $\mathrm{B}$. We also address in Section 3 some crucial field-theoretic issues related to the proper description of a two-level Higgs-boson system strongly mixed via CP violation. Section 4 presents numerical estimates of Higgsboson pole masses, making comparisons with previous results obtained in the RG-improved 
effective-potential approach. Our conclusions are summarized in Section 5 [31.

\section{RG-Improved Higgs-Boson Self-Energies}

In this Section, we describe our approach to calculating the pole masses of the charged and neutral Higgs bosons in the MSSM with explicit radiative CP violation. Our approach utilizes RG methods in the $\overline{\mathrm{MS}}$ scheme developed earlier in [19, 20, 21, 22]. In particular, we demonstrate that, in the limit in which the squared off-shell momentum $s=p^{2}$ of the Higgs-boson propagators goes to zero, our derived analytic results of the Higgs-boson masses are identical to those previously obtained in [12 in the effective Higgs-potential approach.

First we briefly review the basic low-energy structure of the MSSM with explicit CP violation. It is possible for $\mathrm{CP}$ violation to appear via the Higgs superpotential and the soft SUSY-breaking Lagrangian:

$$
\begin{aligned}
W= & h_{l} \widehat{H}_{1}^{T} i \tau_{2} \widehat{L} \widehat{E}+h_{d} \widehat{H}_{1}^{T} i \tau_{2} \widehat{Q} \widehat{D}+h_{u} \widehat{Q}^{T} i \tau_{2} \widehat{H}_{2} \widehat{U}-\mu \widehat{H}_{1}^{T} i \tau_{2} \widehat{H}_{2}, \\
-\mathcal{L}_{\text {soft }}= & -\frac{1}{2}\left(m_{\tilde{g}} \lambda_{\tilde{g}}^{a} \lambda_{\tilde{g}}^{a}+m_{\widetilde{W}} \lambda_{\widetilde{W}}^{i} \lambda_{\widetilde{W}}^{i}+m_{\widetilde{B}} \lambda_{\widetilde{B}} \lambda_{\widetilde{B}}+\text { h.c. }\right)+\widetilde{M}_{L}^{2} \widetilde{L}^{\dagger} \widetilde{L}+\widetilde{M}_{Q}^{2} \widetilde{Q}^{\dagger} \widetilde{Q} \\
& +\widetilde{M}_{U}^{2} \widetilde{U^{*}} \widetilde{U}+\widetilde{M}_{D}^{2} \widetilde{D^{*}} \widetilde{D}+\widetilde{M}_{E}^{2} \widetilde{E}^{*} \widetilde{E}+m_{1}^{2} \widetilde{\Phi}_{1}^{\dagger} \widetilde{\Phi}_{1}+m_{2}^{2} \Phi_{2}^{\dagger} \Phi_{2}-\left(m_{12}^{2} \widetilde{\Phi}_{1}^{T} i \tau_{2} \Phi_{2}\right. \\
& + \text { h.c. })+\left(h_{l} A_{l} \Phi_{1}^{\dagger} \widetilde{L} \widetilde{E}+h_{d} A_{d} \Phi_{1}^{\dagger} \widetilde{Q} \widetilde{D}-h_{u} A_{u} \Phi_{2}^{T} i \tau_{2} \widetilde{Q} \widetilde{U}+\text { h.c. }\right),
\end{aligned}
$$

where $\widetilde{\Phi}_{1}=i \tau_{2} \Phi_{1}^{*}$ is the physical bosonic degree of freedom of the Higgs chiral superfield $\widehat{H}_{1}$, and $\tau_{2}$ is the usual Pauli matrix. Throughout the paper, we follow the conventions of [9, 12]. Details of the notation, including the quantum-number assignments of the fields under the SM gauge group, are given in Table 1 of [12].

The expressions (2.1) and (2.2) contain a large number of new CP-violating phases in the MSSM that are not present in the SM. The large number of these independent CP-odd phases can be substantially reduced if universality conditions are imposed on the complex soft SUSY-breaking parameters of the theory at some high-energy scale $M_{X}$. Under such universality conditions, all soft SUSY-breaking squark masses are set equal to a universal value at the scale $M_{X}$. Similarly, all the soft SUSY-breaking Yukawa coupling coefficients $A_{f}$ are set to a universal value at $M_{X}$, and likewise the gaugino masses $m_{\tilde{g}}, m_{\widetilde{W}}$ and $m_{\widetilde{B}}$ of the gauge groups $\mathrm{SU}(3)_{c}, \mathrm{SU}(2)_{L}$ and $\mathrm{U}(1)_{Y}$ are assumed to share a common value $m_{1 / 2}$ at $M_{X}$. In this unified framework f, the new CP-odd phases are contained in four complex

${ }^{*}$ One should bear in mind that the different RG runnings of the phases of the soft SUSY-breaking Yukawa couplings and of the gaugino masses lead to non-universal values at lower energies [25]. 
parameters: the $\mu$ parameter that mixes the Higgs supermultiplets, the soft bilinear Higgsmixing mass-squared parameter $m_{12}^{2}$, the common gaugino mass $m_{1 / 2}$ and the common soft Yukawa coupling coefficient $A_{f}$. However, two of these four phases may be eliminated using the two global symmetries that govern the dimension-four operators in the MSSM Lagrangian. In this way, the parameters $\mu$ and $m_{12}^{2}$ may be rephased so as to become real numbers. Consequently, the physical CP-violating phases of interest are $\arg \left(A_{f}\right)$ and $\arg \left(m_{1 / 2}\right)$. In this work we shall give general expressions, which may be applicable, but are not restricted, to the case of universal boundary conditions.

The ground state of the radiatively-corrected MSSM Higgs potential $-\mathcal{L}_{V}$ may be determined by first linearly expanding the Higgs doublets $\Phi_{1}$ and $\Phi_{2}$ as

$$
\Phi_{1}=\left(\begin{array}{c}
\phi_{1}^{+} \\
\frac{1}{\sqrt{2}}\left(v_{1}+\phi_{1}+i a_{1}\right)
\end{array}\right), \quad \Phi_{2}=e^{i \xi}\left(\begin{array}{c}
\phi_{2}^{+} \\
\frac{1}{\sqrt{2}}\left(v_{2}+\phi_{2}+i a_{2}\right)
\end{array}\right)
$$

where $v_{1}$ and $v_{2} e^{i \xi}$ are the VEVs of the Higgs doublets, and then demanding that the total tadpole contributions to the neutral CP-even and CP-odd fields $\phi_{1,2}$ and $a_{1,2}$ vanish [8, 9, 12]. If $m_{12}^{2}$ is real at the tree level, then a relative phase $\xi$ of the two VEVs is formally induced at the one-loop level. However, the phase $\xi$ can always be set to zero by a judicious choice of the counter-term (CT) Im $m_{12}^{2}$. Specifically, the CT Im $m_{12}^{2}$ can be chosen in a way such that it cancels completely the CP-odd tadpole graphs, leading to the consistent solution $\xi=0$ for the vanishing of the total CP-odd tadpole contributions. In this context, we stress again $\mathbb{8}$ that such a $\xi=0$ scheme of renormalization preserves the phase convention for $m_{12}^{2}$, and consequently for $\mu$, beyond the Born approximation. It may be extended straightforwardly to all orders in perturbation theory.

It is often more convenient to switch to a basis in which the would-be Goldstone bosons $G^{ \pm}$and $G^{0}$ of the $W^{ \pm}$and $Z$ gauge bosons decouple at $s=p^{2}=0$ from the charged Higgs-boson and pseudoscalar mass matrices, respectively. In such a basis, the new Higgs fields may be expressed in terms of the old ones through the orthogonal rotations:

$$
\left(\begin{array}{c}
G^{+} \\
H^{+}
\end{array}\right)=\left(\begin{array}{cc}
\cos \beta & \sin \beta \\
-\sin \beta & \cos \beta
\end{array}\right)\left(\begin{array}{c}
\phi_{1}^{+} \\
\phi_{2}^{+}
\end{array}\right), \quad\left(\begin{array}{c}
G^{0} \\
a
\end{array}\right)=\left(\begin{array}{cc}
\cos \beta & \sin \beta \\
-\sin \beta & \cos \beta
\end{array}\right)\left(\begin{array}{l}
a_{1} \\
a_{2}
\end{array}\right)
$$

where $\tan \beta \equiv v_{2} / v_{1}$. In (2.4), $H^{+}$and $a$ are the charged and 'CP-odd' neutral Higgs scalars in the effective potential limit: $s \rightarrow 0$. As we see explicitly in the next Section, the effective masses of the physical Higgs-boson fields found in the limit $s \rightarrow 0$ differ from the physical ones determined by the poles of the general propagator matrices. 


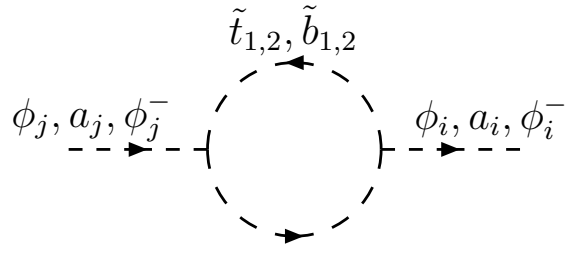

(a)

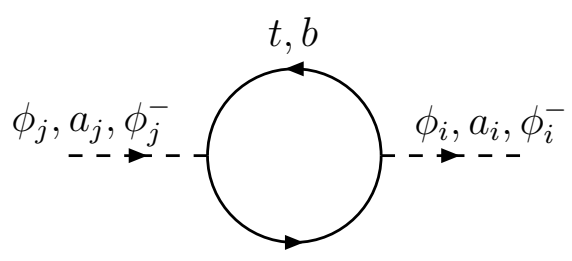

(c)

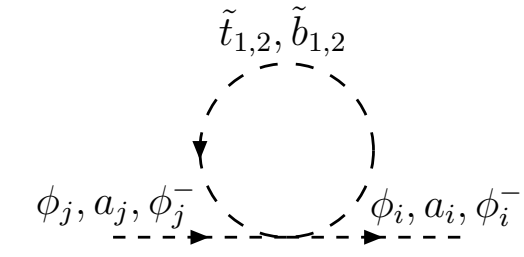

(b)

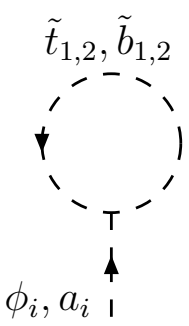

(d)

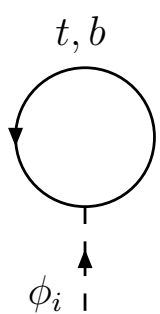

(e)

Figure 1: Third-generation quark and squark contributions to the Higgs-boson self-energies.

We first describe our programme for renormalizing the charged Higgs-boson selfenergies; extending this programme to the neutral Higgs-boson self-energies is then straightforward. We adopt the $\overline{\mathrm{MS}}$ scheme of renormalization. The charged Higgs-boson self-energy transitions $\phi_{j}^{-} \rightarrow \phi_{i}^{-}$, with $i, j=1,2$, span a $2 \times 2$-dimensional matrix, which we denote by $\Pi^{ \pm}(s)$. As shown in Fig. 目, the dominant contributions to $\Pi^{ \pm}(s)$ come from quarks and squarks of the third generation. Employing the couplings of the Higgs bosons to squarks listed in Appendix A, we derive in Appendix B analytic expressions for the unrenormalized charged Higgs-boson self-energies $\Pi^{ \pm}(s)$. Then, in the weak basis $\left\{\phi_{1}^{+}, \phi_{2}^{+}\right\}$, the corresponding renormalized self-energies, denoted by $\widetilde{\Pi}^{ \pm}(s)$, are given by

$$
\widetilde{\Pi}^{ \pm}(s)=\Pi^{ \pm, \overline{\mathrm{MS}}}(s)+\left(\begin{array}{cc}
\frac{T_{\phi_{1}}^{\overline{\mathrm{MS}}}}{v_{1}} & i \frac{T_{a}^{\overline{\mathrm{MS}}}}{v} \\
-i \frac{T_{a}^{\overline{\mathrm{MS}}}}{v} & \frac{T_{\phi_{2}}^{\overline{\mathrm{MS}}}}{v_{2}}
\end{array}\right),
$$

with $T_{a}=T_{a_{2}} / \cos \beta=-T_{a_{1}} / \sin \beta$. In the $\overline{\mathrm{MS}}$ scheme, the renormalized charged Higgsboson self-energy $\Pi^{ \pm, \overline{\mathrm{MS}}}(s)$ is obtained from $\Pi^{ \pm}(s)$ by simply dropping all terms proportional to the ultra-violet (UV) divergence $C_{\mathrm{UV}}$. Such a scheme of renormalization is valid for renormalizable theories, such as the one under study. Similarly, in (2.5), $T_{\phi_{1,2}}^{\overline{\mathrm{MS}}}$ and $T_{a_{1,2}}^{\overline{\mathrm{MS}}}$ are the $\overline{\mathrm{MS}}$-renormalized tadpole CTs as derived from $T_{\phi_{1,2}}$ and $T_{a_{1,2}}$. The latter are computed in Appendix B.

We can now extend the procedure for the RG improvement of effective Higgs-boson mass matrices we presented in [12] to Higgs-boson self-energies. In particular, we wish to 
take properly into account the decoupling properties and threshold effects of the heavy squarks in the loops. Following a line of argument closely related to that in [12], we arrive at the RG-improved charged Higgs-boson self-energy matrix

$$
\widehat{\Pi}_{i j}^{ \pm}(s)=-\left(\overline{\mathcal{M}}_{ \pm}^{2}\right)_{i j}+\left(\xi_{i}^{+} \xi_{j}^{-}\right)^{-1}\left(\Delta \Pi^{ \pm}\right)_{i j}^{\tilde{t} \tilde{b}}(s)+\left(\widetilde{\Pi}^{ \pm}\right)_{i j}^{t b}(s)
$$

Here, $\overline{\mathcal{M}}_{ \pm}^{2}$ is the two-loop Born-improved mass matrix given by

$$
\overline{\mathcal{M}}_{ \pm}^{2}=\mathcal{M}_{ \pm}^{2(0)}\left(\operatorname{Re} \bar{m}_{12}^{2}, \bar{\lambda}_{4}\right)
$$

where

$$
\mathcal{M}_{ \pm}^{2(0)}\left(\operatorname{Re} m_{12}^{2}, \lambda_{4}\right)=\left(\frac{1}{2} \lambda_{4} v_{1} v_{2}+\operatorname{Re} m_{12}^{2}\right)\left(\begin{array}{cc}
\tan \beta & -1 \\
-1 & \cot \beta
\end{array}\right)
$$

is the tree-level charged Higgs-boson mass matrix expressed in terms of relevant parameters such as the real part of the soft bilinear Higgs mixing, Re $m_{12}^{2}$, and the quartic coupling $\lambda_{4}$. The bar on these parameters in (2.7), i.e. Re $\bar{m}_{12}^{2}$ and $\bar{\lambda}_{4}$, indicates the sum of the tree-level and of the one- and two-loop leading logarithmic contributions. The analytic forms of $\operatorname{Re} \bar{m}_{12}^{2}$ and $\bar{\lambda}_{1} \ldots \bar{\lambda}_{4}$ are given in [12].

The second term in (2.6) describes the threshold effect of the top and bottom squarks and is the product of two quantities: (i) the anomalous-dimension factors of the external Higgs fields

$$
\xi_{1}^{ \pm}=\xi_{1}^{ \pm}\left(m_{t}\right)=1+\frac{3\left|h_{b}\right|^{2}}{32 \pi^{2}} \ln \frac{Q_{t b}^{2}}{m_{t}^{2}}, \quad \xi_{2}^{ \pm}=\xi_{2}^{ \pm}\left(m_{t}\right)=1+\frac{3\left|h_{t}\right|^{2}}{32 \pi^{2}} \ln \frac{Q_{t b}^{2}}{m_{t}^{2}},
$$

where $Q_{t b}^{2}=\max \left(\widetilde{M}_{Q}^{2}+m_{t}^{2}, \widetilde{M}_{t}^{2}+m_{t}^{2}, \widetilde{M}_{b}^{2}+m_{b}^{2}\right)$ defines the decoupling scale of the heavy squarks, and (ii) the threshold contribution

$$
\Delta \Pi^{ \pm}(s)=\mathcal{M}_{ \pm}^{2(0)}\left(\operatorname{Re} m_{12}^{2(1)}, \lambda_{4}^{(1)}\right)+\widetilde{\Pi}^{ \pm}(s)
$$

which is manifestly scale-independent at the one-loop level. The superscript '(1)' attached to the quantities $\operatorname{Re} m_{12}^{2}$ and $\lambda_{1} \ldots \lambda_{4}$ indicates one-loop leading logarithmic contributions. Finally, the third term in (2.6) includes the top- and bottom-quark effects on the charged Higgs-boson self-energies.

Like any quantity calculated in perturbation theory, the Higgs-boson mass spectrum is subject to an uncertainty related to unknown higher-order contributions, which is reflected in arbitrariness associated with the choice of renormalization scale. In making this choice, one may use as a guiding principle the minimization of the two-loop corrections in the effective-potential limit $s \rightarrow 0$ [20, 21]. Since for sparticle masses above the weak scale 
this minimization was shown to occur close to the top-quark mass $m_{t}$ [20, 21], we perform our computations using this renormalization scale. Note that in the limit $s \rightarrow 0$, the RGimproved charged Higgs-boson mass matrix $\mathcal{M}_{ \pm}^{2}$ and the corresponding self-energy matrix $\widehat{\Pi}^{ \pm}(s)$ are related by $\mathcal{M}_{ \pm}^{2}=-\widehat{\Pi}^{ \pm}(s=0)$.

The neutral Higgs-boson self-energies can be computed following a path very analogous to the charged Higgs-boson case. First, we compute the $\overline{\mathrm{MS}}$-renormalized, but not RG-improved, Higgs-boson self-energies that involve the transitions $\phi_{j} \rightarrow \phi_{i}, \phi_{j} \rightarrow a_{i}$ and $a_{j} \rightarrow a_{i}$, with $i, j=1,2$. These $\overline{\mathrm{MS}}$-renormalized scalar-scalar, scalar-pseudoscalar and pseudoscalar-pseudoscalar transitions are given by the two-by-two self-energy matrices:

$$
\begin{aligned}
\widetilde{\Pi}^{S}(s) & =\Pi^{S, \overline{\mathrm{MS}}}(s)+\operatorname{diag}\left(\frac{T_{\phi_{1}}^{\overline{\mathrm{MS}}}}{v_{1}}, \frac{T_{\phi_{2}}^{\overline{\mathrm{MS}}}}{v_{2}}\right), \\
\widetilde{\Pi}^{S P}(s) & =\Pi^{S P, \overline{\mathrm{MS}}}(s)+i \tau_{2} \frac{T_{a}^{\overline{\mathrm{MS}}}}{v}, \\
\widetilde{\Pi}^{P}(s) & =\Pi^{P, \overline{\mathrm{MS}}}(s)+\operatorname{diag}\left(\frac{T_{\phi_{1}}^{\overline{\mathrm{MS}}}}{v_{1}}, \frac{T_{\phi_{2}}^{\overline{\mathrm{MS}}}}{v_{2}}\right),
\end{aligned}
$$

where the corresponding unrenormalized self-energies $\Pi^{S}(s), \Pi^{S P}(s)$ and $\Pi^{P}(s)$ may be found in Appendix B. Then, very analogously with the charged Higgs-boson case (2.6), we carry out the RG improvement of the neutral Higgs-boson self-energies by considering the squark threshold effects, i.e. ,

$$
\widehat{\Pi}_{i j}^{S}(s)=-\left(\overline{\mathcal{M}}_{S}^{2}\right)_{i j}+\sum_{\tilde{q}=\tilde{t}, b}\left(\xi_{i}^{\tilde{q}} \xi_{j}^{\tilde{q}}\right)^{-1}\left(\Delta \Pi^{S}\right)_{i j}^{\tilde{q}}(s)+\left(\widetilde{\Pi}^{S}\right)_{i j}^{t b}(s)
$$

and likewise for $\widehat{\Pi}^{S P}(s)$ and $\widehat{\Pi}^{P}(s)$. As in the charged Higgs-boson case, we denote by $\overline{\mathcal{M}}_{S}^{2}$ in (2.12) the CP-even two-loop Born-improved mass matrix and by $\xi_{i}^{\tilde{q}}$ the anomalousdimension factors

$$
\begin{array}{ll}
\xi_{1}^{\tilde{t}}\left(m_{t}\right)=1+\frac{3\left|h_{b}\right|^{2}}{32 \pi^{2}} \ln \frac{Q_{t}^{2}}{m_{t}^{2}}, & \xi_{2}^{\tilde{t}}\left(m_{t}\right)=1+\frac{3\left|h_{t}\right|^{2}}{32 \pi^{2}} \ln \frac{Q_{t}^{2}}{m_{t}^{2}} \\
\xi_{1}^{\tilde{b}}\left(m_{t}\right)=1+\frac{3\left|h_{b}\right|^{2}}{32 \pi^{2}} \ln \frac{Q_{b}^{2}}{m_{t}^{2}}, & \xi_{2}^{\tilde{b}}\left(m_{t}\right)=1+\frac{3\left|h_{t}\right|^{2}}{32 \pi^{2}} \ln \frac{Q_{b}^{2}}{m_{t}^{2}}
\end{array}
$$

which appropriately define the running from the squark-decoupling scales $Q_{t}^{2}=\max \left(\widetilde{M}_{Q}^{2}+\right.$ $\left.m_{t}^{2}, \widetilde{M}_{t}^{2}+m_{t}^{2}\right)$ and $Q_{b}^{2}=\max \left(\widetilde{M}_{Q}^{2}+m_{b}^{2}, \widetilde{M}_{b}^{2}+m_{b}^{2}\right)$ down to the top-quark mass $m_{t}$.

${ }^{*}$ This procedure involves the approximation of identifying the quark Yukawa couplings with the squark ones even below the SUSY-breaking scale. An explicit comparison of the effective potential computation with the diagrammatic two-loop computation of the lightest Higgs-boson mass shows that, even for the extreme conditions analyzed in [26], the numerical differences between both methods remain of about $2.5 \mathrm{GeV}$ [27]. 
In addition, $\left(\Delta \Pi^{S}\right)^{\tilde{t}, \tilde{b}}(s)$ is the one-loop scale-independent contribution induced by the top and bottom squarks individually. Finally, $\left(\widetilde{\Pi}^{S}\right)^{t b}(s)$ represents the top- and bottom-quark contributions to the CP-even Higgs-boson self-energies.

The same procedure may be used to obtain the RG-improved analytic forms of the remaining neutral Higgs-boson self-energies: $\widehat{\Pi}^{S P}(s)$ and $\widehat{\Pi}^{P}(s)$. Thus, in the weak basis $\left\{\phi_{1}, \phi_{2}, a_{1}, a_{2}\right\}$, all the RG-improved neutral Higgs-boson self-energies may be summarized by the $4 \times 4$-dimensional matrix

$$
\widehat{\Pi}^{N}(s)=\left(\begin{array}{cc}
\widehat{\Pi}^{S}(s) & \widehat{\Pi}^{S P}(s) \\
\left(\widehat{\Pi}^{S P}(s)\right)^{T} & \widehat{\Pi}^{P}(s)
\end{array}\right) .
$$

In the effective-potential limit $s \rightarrow 0$, the would-be Goldstone bosons $G^{ \pm}$and $G^{0}$ defined in (2.4) decouple from the charged and neutral Higgs-boson matrices. As a consequence, the ranks of the respective self-energy matrices given by (2.6) and (2.14) get reduced by one unit. To make this last property explicit, we express the RG-improved charged and neutral Higgs-boson self-energies $\widehat{\Pi}^{ \pm}(s)$ and $\widehat{\Pi}^{N}(s)$ in the weak bases $\left\{G^{+}, H^{+}\right\}$and $\left\{\phi_{1}, \phi_{2}, a, G^{0}\right\}$, respectively. In the following we assume that such a change of weak bases has already been made using (2.4).

It is worth noticing that the RG-improved charged and neutral Higgs-boson selfenergies include, through the terms $\Delta \Pi^{ \pm}, \Delta \Pi^{S}$, etc., potentially large two-loop nonlogarithmic contributions from third-generation squarks, which are induced by their oneloop threshold effects on the top- and bottom-quark Yukawa couplings $h_{t}$ and $h_{b}$. Since these effects were extensively studied in [12 within the MSSM with explicit radiative CP violation, we do not embark on this topic here as well. In the next section, instead, we calculate analytically the pole masses of the charged and neutral Higgs bosons, and discuss some field-theoretic subtleties relevant to this calculation.

\section{Higgs-Boson Pole Masses}

We now concentrate on field-theoretic issues related to the proper definition of the physical pole masses of the unstable charged and neutral Higgs bosons. In the MSSM with explicit radiative $\mathrm{CP}$ violation, as well as being almost degenerate in mass, the two heaviest neutral Higgs bosons may mix with one another very strongly. This is a novel feature absent from the CP-invariant MSSM [8]. In particular, a proper description of the strong CP-violating mixing of the heavier Higgs bosons requires a resummation approach that takes properly into account unstable particle-mixing effects [28] in a way consistent with unitarity and 
gauge invariance. For this, one might consider the resummation approach implemented by the pinch technique (PT) [29]. In general, when naively resummed, off-shell Higgs-boson self-energies depend on the choice of the gauge-fixing parameter, due to loops involving $W^{ \pm}$and $Z$ gauge bosons. However, this issue of gauge invariance does not directly affect the present computation of the Higgs-boson pole masses, since we neglect the subdominant contributions due to gauge-boson loops. Moreover, it is known [30] that pole masses are by themselves gauge-invariant quantities.

To start with, we consider the charged Higgs-boson sector. The dispersion of the charged Higgs fields is governed by the inverse propagator matrix

$$
\hat{\Delta}_{ \pm}^{-1}(s)=s \mathbf{1}_{2}+\widehat{\Pi}^{ \pm}(s)
$$

where $\widehat{\Pi}^{ \pm}(s)$ is the RG-improved self-energy matrix of the charged Higgs fields expressed in the weak basis $\left\{G^{ \pm}, H^{ \pm}\right\}$. Note that $\widehat{\Pi}^{ \pm}(s)$ defined in (2.6) includes the tree-level contributions and so does $\hat{\Delta}_{ \pm}(s)$. Therefore, it is more convenient to rewrite $\widehat{\Pi}^{ \pm}(s)$ as

$$
\widehat{\Pi}^{ \pm}(s)=\left(\begin{array}{cc}
\widehat{\Pi}_{G^{+} G^{-}}(s) & \widehat{\Pi}_{G^{+} H^{-}}(s) \\
\widehat{\Pi}_{H^{+} G^{-}}(s) & -M_{H^{+}}^{2(0)}+\widehat{\Pi}_{H^{+} H^{-}}(s)
\end{array}\right),
$$

where $M_{H^{+}}^{2(0)}$ is the mass eigenvalue of the tree-level charged Higgs-boson mass matrix $\mathcal{M}_{ \pm}^{2(0)}$ given by (2.8). The gauge-independent pole masses of the $G^{+}-H^{+}$system are determined by the vanishing of $\operatorname{det} \hat{\Delta}_{ \pm}^{-1}(s)$. Thus, the complex pole masses $\hat{s}_{G^{+}}$and $\hat{s}_{H^{+}}$of the $G^{+}$and $H^{+}$bosons are found to be

$$
\begin{aligned}
\hat{s}_{G^{+}\left(H^{+}\right)} & =\widehat{M}_{G^{+}\left(H^{+}\right)}^{2}-i \widehat{M}_{G^{+}\left(H^{+}\right)} \widehat{\Gamma}_{G^{+}\left(H^{+}\right)} \\
& =-\frac{1}{2}\left[\operatorname{Tr} \widehat{\Pi}^{ \pm}\left(\hat{s}_{G^{+}\left(H^{+}\right)}\right)-(+) \sqrt{\operatorname{Tr}^{2} \widehat{\Pi}^{ \pm}\left(\hat{s}_{G^{+}\left(H^{+}\right)}\right)-4 \operatorname{det} \widehat{\Pi}^{ \pm}\left(\hat{s}_{G^{+}\left(H^{+}\right)}\right)}\right]
\end{aligned}
$$

where $\widehat{\Gamma}_{G^{+}\left(H^{+}\right)}$correspond to the width of the corresponding particles. Employing the fact that $\operatorname{det} \widehat{\Pi}^{ \pm}(0)=0$ implies $\hat{s}_{G^{+}}=0$, as follows from the Goldstone theorem. Since it is practically impossible to solve (3.3) analytically for $\hat{s}_{H^{+}}$, we shall determine the pole mass of the charged Higgs boson $H^{+}$using a perturbative expansion with respect to the loop factors $1 /\left(16 \pi^{2}\right)^{n}$, where $n$ indicates the number of loops. To this end, we consider the relation

$$
\hat{s}_{H^{+}}=M_{H^{+}}^{2(0)}-\widehat{\Pi}_{H^{+} H^{-}}\left(\hat{s}_{H^{+}}\right)+\frac{\widehat{\Pi}_{H^{+} G^{-}}\left(\hat{s}_{H^{+}}\right) \widehat{\Pi}_{G^{+} H^{-}}\left(\hat{s}_{H^{+}}\right)}{\hat{s}_{H^{+}}+\widehat{\Pi}_{G^{+} G^{-}}\left(\hat{s}_{H^{+}}\right)},
$$

which is completely equivalent to (3.3). Within the framework of perturbation theory, a self-energy function $\Pi(s)$, e.g. $\widehat{\Pi}_{H^{+} H^{-}}(s)$, may be expanded loopwise as

$$
\Pi(s)=\Pi^{(1)}(s)+\Pi^{(2)}(s)+\cdots+\Pi^{(n)}(s)+\cdots .
$$


Obviously, at zeroth order we have $\widehat{M}_{H^{+}}^{2(0)}=M_{H^{+}}^{2(0)}$ and $\widehat{\Gamma}_{H^{+}}^{(0)}=0$, and at one-loop order $\widehat{M}_{H^{+}}^{2(1)}=\widehat{M}_{H^{+}}^{2(0)}-\operatorname{Re} \widehat{\Pi}_{H^{+} H^{-}}^{(1)}\left(\widehat{M}_{H^{+}}^{2(1)}\right)$ and $\widehat{M}_{H^{+}}^{(1)} \widehat{\Gamma}_{H^{+}}^{(1)}=\operatorname{Im} \widehat{\Pi}_{H^{+} H^{-}}^{(1)}\left(\widehat{M}_{H^{+}}^{2(1)}\right)$. Making use of the emerging perturbative structure, we find at two-loop order

$$
\begin{aligned}
\widehat{M}_{H^{+}}^{2(2)}= & \widehat{M}_{H^{+}}^{2(0)}-\operatorname{Re}\left(\widehat{\Pi}_{H^{+} H^{-}}^{(1)}+\widehat{\Pi}_{H^{+} H^{-}}^{(2)}\right)-\operatorname{Im} \widehat{\Pi}_{H^{+} H^{-}}^{(1) \prime} \operatorname{Im} \widehat{\Pi}_{H^{+} H^{-}}^{(1)} \\
& +\frac{1}{\widehat{M}_{H^{+}}^{2(2)}} \operatorname{Re}\left(\widehat{\Pi}_{H^{+} G^{-}}^{(1)} \widehat{\Pi}_{G^{+} H^{-}}^{(1)}\right), \\
\widehat{M}_{H^{+}}^{(2)} \widehat{\Gamma}_{H^{+}}^{(2)}= & \operatorname{Im}\left(\widehat{\Pi}_{H^{+} H^{-}}^{(1)}+\widehat{\Pi}_{H^{+} H^{-}}^{(2)}\right)-\operatorname{Re} \widehat{\Pi}_{H^{+} H^{-}}^{(1) \prime} \operatorname{Im} \widehat{\Pi}_{H^{+} H^{-}}^{(1)} \\
& -\frac{1}{\widehat{M}_{H^{+}}^{2(2)}} \operatorname{Im}\left(\widehat{\Pi}_{H^{+} G^{-}}^{(1)} \widehat{\Pi}_{G^{+} H^{-}}^{(1)}\right) .
\end{aligned}
$$

Here, all self-energies involved are understood to be evaluated at $\widehat{M}_{H^{+}}^{2(2)}$. Since we are interested in a perturbative one-loop computation of the charged Higgs-boson pole mass that is improved by including two-loop leading logarithmic corrections and threshold effects, we should consistently disregard higher-order terms occurring in $\widehat{M}_{H^{+}}^{(2)}$. Specifically, since the third and fourth terms on the right-hand side of (3.6) do not receive leading logarithmic contributions, they may be omitted [.

In the MSSM with explicit radiative CP violation, it is convenient to use as input the physical charged Higgs-boson mass $\widehat{M}_{H^{+}}$instead of Re $\bar{m}_{12}^{2}$ in (2.7). The free Lagrangian parameter Re $\bar{m}_{12}^{2}$ also enters the neutral Higgs-boson self-energy matrix $\widehat{\Pi}^{N}(s)$ through the two-loop Born-improved mass matrix $\overline{\mathcal{M}}_{S}^{2}$ in (2.12) (and likewise $\overline{\mathcal{M}}_{P}^{2}$ ). We may therefore eliminate the quantity $\operatorname{Re} \bar{m}_{12}^{2}$ appearing in $\widehat{\Pi}^{N}(s)$ in favour of $\widehat{M}_{H^{+}}^{2}$, using the relation

$$
\frac{\operatorname{Re} \bar{m}_{12}^{2}}{\sin \beta \cos \beta}=\widehat{M}_{H^{+}}^{2}-\frac{1}{2} \bar{\lambda}_{4} v^{2}+\operatorname{Re} \widehat{\Pi}_{H^{+} H^{-}}\left(\widehat{M}_{H^{+}}^{2}\right) .
$$

In (3.8), all kinematic parameters, with the exception of the scale-independent quantity $\widehat{M}_{H^{+}}^{2}$, are evaluated at $m_{t}$. The charged Higgs-boson self-energy $\widehat{\Pi}_{H^{+} H^{-}}(s)$ is calculated to the one-loop order considered, and further improved by including leading two-loop logarithms and threshold effects.

The computation of the pole masses of the neutral Higgs bosons can proceed along similar lines. As in the charged Higgs-boson case, to the order of our perturbative approximation, we may neglect all the self-energy terms involving the $G^{0}$ boson in $\widehat{\Pi}^{N}$, i.e. the terms $\widehat{\Pi}_{G^{0} G^{0}}, \widehat{\Pi}_{G^{0} a}, \widehat{\Pi}_{G^{0} \phi_{1}}$ and $\widehat{\Pi}_{G^{0} \phi_{2}}$. However, we cannot neglect the other matrix elements of $\widehat{\Pi}^{N}$ in the subspace $\left\{\phi_{1}, \phi_{2}, a\right\}$, and especially those induced by CP-violating loop effects.

\footnotetext{
${ }^{\dagger}$ Moreover, we have verified in a number of cases that these higher-order terms indeed have negligible numerical impact on the neutral Higgs-boson mass spectrum.
} 
To better elucidate the above point, let us consider two Higgs scalars $H$ and $A$ of opposite $\mathrm{CP}$ quantum numbers: $\mathrm{CP}(H)=+1$ and $\mathrm{CP}(A)=-1$. For instance, for $\tan \beta \gtrsim 3$ and $M_{H^{+}} \gtrsim 200 \mathrm{GeV}$, one may identify $H$ approximately with $\phi_{1}$ and $A$ with $a$, the two heaviest neutral Higgs states in the CP-invariant limit of the theory. Further, the states $H$ and $A$ may be considered to be nearly degenerate, which is exactly what happens in the CP-invariant MSSM for the above kinematic region of a large $M_{H^{+}}$. In the presence of explicit sources of $\mathrm{CP}$ violation (see also the discussion in Section 2), $H$ and $A$ will mix radiatively, and one then has to deal with a strong $\mathrm{CP}$-violating $H$ - $A$ mixing system [8, 28]. The dynamics of the $H$ - $A$ system may be described by the two-by-two propagator matrix $\hat{\Delta}_{n}(s)$, whose inverse is given by

$$
\hat{\Delta}_{n}^{-1}(s)=s \mathbf{1}_{2}+\widehat{\Pi}^{n}(s)=s \mathbf{1}_{2}-\left(\begin{array}{cc}
M_{H}^{2(0)}-\widehat{\Pi}_{H H}(s) & -\widehat{\Pi}_{H A}(s) \\
-\widehat{\Pi}_{A H}(s) & M_{A}^{2(0)}-\widehat{\Pi}_{A A}(s)
\end{array}\right) .
$$

As before, the pole masses $\hat{s}_{H}$ and $\hat{s}_{A}$ of the $H$ and $A$ bosons may be obtained as solutions of the characteristic equation $\operatorname{det} \hat{\Delta}_{n}^{-1}(s)=0$. The last equation can equivalently be decomposed into a coupled system of complex equations:

$$
\begin{aligned}
& \hat{s}_{H}=M_{H}^{2(0)}-\widehat{\Pi}_{H H}\left(\hat{s}_{H}\right)+\frac{\widehat{\Pi}_{H A}\left(\hat{s}_{H}\right) \widehat{\Pi}_{A H}\left(\hat{s}_{H}\right)}{\hat{s}_{H}-M_{A}^{2(0)}+\widehat{\Pi}_{A A}\left(\hat{s}_{H}\right)}, \\
& \hat{s}_{A}=M_{A}^{2(0)}-\widehat{\Pi}_{A A}\left(\hat{s}_{A}\right)+\frac{\widehat{\Pi}_{A H}\left(\hat{s}_{A}\right) \widehat{\Pi}_{H A}\left(\hat{s}_{A}\right)}{\hat{s}_{A}-M_{H}^{2(0)}+\widehat{\Pi}_{H H}\left(\hat{s}_{A}\right)} .
\end{aligned}
$$

Within the framework of perturbation theory, the above non-linear system of equations could be solved iteratively, where $\hat{s}_{H}$ and $\hat{s}_{A}$ on the left-hand sides of (3.10) and (3.11) represent higher degrees of iteration. Such a procedure, however, does not lead to convergent solutions when

$$
\left|M_{H}^{2(0)}-\widehat{\Pi}_{H H}(s)-M_{A}^{2(0)}+\widehat{\Pi}_{A A}(s)\right| \lesssim 2\left|\widehat{\Pi}_{H A}(s)\right|
$$

at $s \approx \widehat{M}_{H}^{2} \approx \widehat{M}_{A}^{2}$. Here, it is also interesting to remark that the inequality (3.12) reflects a kinematic regime of resonantly-enhanced CP violation in Higgs-mediated processes [28].

To avoid the above problem of convergence in the computation of pole masses, we follow a different two-step approach, which we apply directly to the original $4 \times 4$ self-energy matrix $\widehat{\Pi}^{N}(s)$. The first step consists in diagonalizing $\Pi^{N}(s)$ in the effective-potential limit $s=0$ by means of an orthogonal matrix $O$ :

$$
O^{T} \widehat{\Pi}^{N}(s) O=\bar{\Pi}^{N}(s)
$$


with

$$
-\bar{\Pi}^{N}(s=0)=\operatorname{diag}\left(M_{H_{1}}^{2}, M_{H_{2}}^{2}, M_{H_{3}}^{2}, 0\right) .
$$

Clearly, the analytic forms of $M_{H_{1,2,3}}$ coincide with the RG-improved Higgs-boson masses computed in [12]. In particular, we can check that the numerical results obtained by the two approaches are identical, provided the effective-potential charged Higgs-boson mass $M_{H^{+}}$ instead of the pole mass $\widehat{M}_{H^{+}}$is used as an input. In the second step, we determine the perturbative one-loop pole masses $\widehat{M}_{H_{1,2,3}}^{2}$ of the three Higgs bosons through the relation:

$$
\widehat{M}_{H_{i}}^{2}=-\operatorname{Re} \bar{\Pi}_{i i}^{N}\left(M_{H_{i}}^{2}\right) \text {, }
$$

with $i=1,2,3$.

Clearly, the above expression is an excellent approximation to the exact masses in cases in which the mass eigenvalues are not close to each other. For nearly degenerate Higgs bosons which are strongly mixed by $\mathrm{CP}$ violation, e.g. for $\left|M_{H_{2}}^{2}-M_{H_{3}}^{2}\right| \lesssim \mid \bar{\Pi}_{23}^{N}\left(M_{H_{2}}^{2}\right)+$ $\bar{\Pi}_{23}^{N}\left(M_{H_{3}}^{2}\right) \mid$, our two-step computation of the Higgs-boson pole masses should be further improved. Indeed, this situation is equivalent to the one we encountered above for a strongly-mixed $H$ - $A$ system (cf. (3.12)). In this case, we first define an average Higgsboson mass, $M_{H_{23}}^{2}=\frac{1}{2}\left(M_{H_{2}}^{2}+M_{H_{3}}^{2}\right)$, and then find the mass eigenvalues of the two-by-two

submatrix $\bar{\Pi}_{i j}^{N}\left(M_{H_{23}}^{2}\right)$ (with $\left.i, j=2,3\right)$, where the $\left(H_{2}, H_{3}\right)$ subspace is described by a formula analogous to (3.3). In this case, however, all expressions are defined at the scale $M_{H_{23}}^{2}$ and therefore the appropriate solutions may be found in a straightforward way.

\section{Numerical results}

For our quantitative analysis [31], we adopt the benchmark scenario of maximal CP violation $(\mathrm{CPX})$ introduced in [16]:

$$
\begin{aligned}
& \widetilde{M}_{Q}=\widetilde{M}_{t}=\widetilde{M}_{b}=M_{\mathrm{SUSY}}, \quad \mu=4 M_{\mathrm{SUSY}}, \\
& \left|A_{t}\right|=\left|A_{b}\right|=2 M_{\mathrm{SUSY}}, \quad\left|m_{\tilde{g}}\right|=1 \mathrm{TeV} .
\end{aligned}
$$

According to our phase conventions, the parameters $\mu$ and $m_{12}^{2}$ are taken to be real, whilst the soft-trilinear Yukawa couplings $A_{t, b}$ and gluino mass $m_{\tilde{g}}$ are complex. Thus, CP violation in the MSSM Higgs sector is predominantly mediated by the CP-odd phase $\arg \left(A_{t, b}\right)$ at the one-loop level, with $\arg \left(m_{\tilde{g}}\right)$ entering at the two-loop level.

We recall that the CP-violating parameters $A_{t}$ and $m_{\tilde{g}}$ may also lead to observable two- and three-loop contributions to the electron and neutron electric dipole moments 
(EDMs) 32, 33, 34, 35]. Since these higher-loop EDM effects get enhanced for large values of $\tan \beta$, cancellations among the different one- and higher-loop EDM terms may be needed [36, 12]. A cancellation down to the required $10 \%$ level is generally not very difficult to arrange in the CPX scenario, even for relatively large values of $\tan \beta \approx 20$.

As was already discussed in [16], the large values of $|\mu|$ together with the comparatively low values of the charged Higgs-boson mass that are used below in the analysis of the CPX scenario are not feasible in minimal supergravity models with radiative electroweak symmetry breaking. In general, the realization of the CPX scenario requires non-standard boundary conditions at the high-energy input scale. Nevertheless, these non-standard boundary conditions might be obtained, for instance, within superstring-inspired models, in which SUSY is broken by the VEV's of the auxiliary components of the moduli fields.

As a first example, we consider a CPX scenario with $\tan \beta=5, \widehat{M}_{H^{+}}=150 \mathrm{GeV}$ and $M_{\mathrm{SUSY}}=0.5 \mathrm{TeV}$. In Fig. 2(a), we show numerical values for the effective-potential and pole masses of the two lightest Higgs bosons $H_{1}$ and $H_{2}$ as functions of $\arg \left(A_{t}\right)=\arg \left(A_{b}\right)$, for two different gluino phases: $\arg \left(m_{\tilde{g}}\right)=0$ and $90^{\circ}$. In detail, the numerical estimates of the effective-potential masses $M_{H_{1}}$ and $M_{H_{2}}$ are indicated by solid lines for $\arg \left(m_{\tilde{g}}\right)=0$, and by dash-dotted lines for $\arg \left(m_{\tilde{g}}\right)=90^{\circ}$. The corresponding numerical results for the pole masses $\widehat{M}_{H_{1}}$ and $\widehat{M}_{H_{2}}$ are given by the dashed and dotted lines. As can be seen from Fig. 2(a), the difference between effective potential and pole masses becomes significant only for large values of the stop mixing $\left|X_{t}\right|=\left|A_{t}-\mu^{*} / \tan \beta\right|$. This difference can be as large as $3 \mathrm{GeV}$, for $\arg \left(A_{t}\right) \approx \pm 90^{\circ}$.

One may observe that for $\arg \left(m_{\tilde{g}}\right)=0^{\circ}$, the numerical values for the $H_{1^{-}}$and $H_{2^{-}}$ boson masses are approximately symmetric around the vertical line $\arg \left(A_{t}\right)=0$, whilst they exhibit a noticeable asymmetry if $\arg \left(m_{\tilde{g}}\right)=90^{\circ}$. This asymmetry between positive and negative values of $\arg \left(A_{t}\right)$ may be as large as $3 \mathrm{GeV}$. Its origin can be attributed to the CP-violating stop threshold effects on the top-quark Yukawa coupling. The main effect of these threshold corrections is to change the absolute value of the top-quark Yukawa coupling at the heavy squark-mass scale, which determines the value of the scale-independent contributions to the effective potential. Taking into account the expression for $h_{t}$ presented in [12], the dominant contribution to these threshold corrections is proportional to $A_{t} m_{\tilde{g}}$.

Since the tree-level top quark Yukawa coupling is real, for zero gluino-mass phase, a change in sign of the phase of $A_{t}$ leads to a change in sign of the imaginary part of the threshold correction, without inducing any change in the absolute value of the top quark Yukawa coupling at the one-loop level. For $\arg \left(m_{\tilde{g}}\right)=90^{\circ}$, instead, choosing $\arg \left(A_{t}\right)=$ $90^{\circ}\left(-90^{\circ}\right)$ leads to a threshold correction that reduces (enhances) the absolute value of 
$h_{t}$. Indeed, the product of $A_{t}$ and $m_{\tilde{g}}$ reduces to a negative (positive) real number for this particular choice of phases and therefore the situation is similar to the CP-conserving case in which positive (negative) values of $A_{t}$ enhance (reduce) $\left|h_{t}\right|$. As is clear from Fig. 2(a), an increase (reduction) of the top quark Yukawa couplings leads to a corresponding increase (reduction) of the lightest Higgs-boson mass. In this respect, it is interesting to notice that the numerical values for $\arg \left(m_{\tilde{g}}\right)=-90^{\circ}$, which were not plotted in Fig. 2(a), exhibit an asymmetry as well. In fact, the numerical lines for $\arg \left(m_{\tilde{g}}\right)=-90^{\circ}$ are almost mirrorsymmetric with respect to those for $\arg \left(m_{\tilde{g}}\right)=90^{\circ}$ around the $\arg \left(A_{t}\right)=0$ line, in agreement with what is expected from the argument presented above.

In Fig. 2(b), we present numerical estimates of the effective Higgs- $Z-Z$ couplings squared $g_{H_{i} Z Z}^{2}$ (with $\left.i=1,2,3\right)$ versus $\arg \left(A_{t}\right)=\arg \left(A_{b}\right)$, for the above two choices of the gluino phase, i.e. for $\arg \left(m_{\tilde{g}}\right)=0$ and $90^{\circ}$. Note that $g_{H_{i} Z Z}$ represent the effective Higgs couplings to the $Z$ boson normalized to their SM value; the precise determination of $g_{H_{i} Z Z}$ in terms of the orthogonal Higgs-mixing matrix $O$ may be found in [9, 12, 16]. From Fig. 2(b), we see that the predictions for $g_{H_{i} Z Z}^{2}$ are affected in a minor way by the value of the gluino phase.

In Fig. 3, we show numerical estimates for the $H_{1}$ - and $H_{2}$-boson masses and for the effective Higgs couplings to the $Z$ boson in a CPX scenario with $M_{\mathrm{SUSY}}=1 \mathrm{TeV}$. Again, we observe very similar features as in Fig. 2, namely the difference between the effectivepotential and pole masses may be as large as $3 \mathrm{GeV}$, while there is no observable difference in the effective Higgs- $Z-Z$ couplings.

The above results show that the differences between the pole masses and those previously computed in the effective-potential approximation for light neutral Higgs bosons are small, of about the same size of the possible uncertainties coming from higher-order loop corrections. The effects become more relevant in the case of a heavy and stronglymixed Higgs sector, like that obtained in the CPX scenario for relatively large values of the charged Higgs-boson mass.

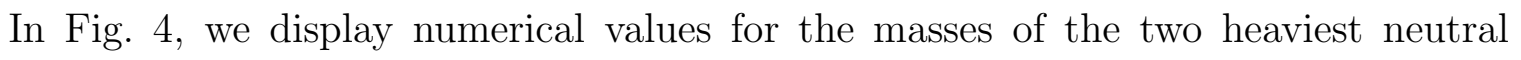
Higgs bosons, $H_{2}$ and $H_{3}$, as functions of $\arg \left(A_{t}\right)$, in a CPX scenario with $\tan \beta=5$, $M_{\mathrm{SUSY}}=0.5 \mathrm{TeV}$ and $\arg \left(m_{\tilde{g}}\right)=90^{\circ}$. Going from the upper to the lower panel in Fig. 4, we discretely vary the charged Higgs-boson pole mass: $\widehat{M}_{H^{+}}=200,400,600 \mathrm{GeV}$. Numerical results pertaining to the effective-potential masses are indicated by solid lines, while the results of pole masses are given by the dashed lines.

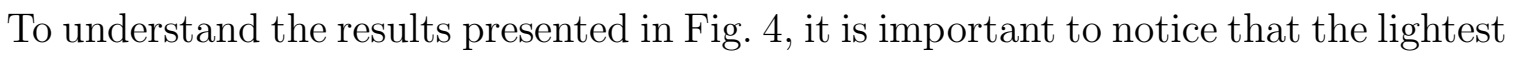
stop mass values in the CPX scenario for $\tan \beta=5$ and $M_{\mathrm{SUSY}}=0.5 \mathrm{TeV}$ vary from 250 to 
$450 \mathrm{GeV}$, with values diminishing for larger values of the phase of $A_{t}$. Whilst a light Higgs boson cannot decay into a pair of supersymmetric particles, this decay channel opens up for a sufficiently heavy charged Higgs boson. An enhancement in the Higgs-boson polemass shift for Higgs-boson masses above the stop production threshold is to be expected if. This effect is quite visible in Fig. 4 . The pole masses start presenting significant differences from those computed in the effective-potential approximation, with the maximal differences observed at the values of the Higgs-boson masses equal to the threshold for the production of a pair of top squarks. This threshold is quite visible in Fig. 4 , for which sizeable mass differences of about ten percent of the Higgs-boson mass are obtained. In particular, this is clearly shown in the cases of $\widehat{M}_{H^{+}}=600 \mathrm{GeV}$, for which mass shifts of about $50 \mathrm{GeV}$ are obtained $\mathrm{f}$. We note that the threshold effect on the $H_{3}$ mass is much smaller than on that of $H_{2}$, due to a significantly smaller coupling of the $H_{3}$ to the lighter stop quarks in the parameter range studied.

In this respect, it is instructive to compare Fig. 1 with Fig. 5. Whilst in Fig. 田 we considered $M_{\mathrm{SUSY}}=0.5 \mathrm{TeV}$, in Fig. 5 we considered $M_{\mathrm{SUSY}}=1 \mathrm{TeV}$. Contrary to the case of a light Higgs sector, in the case of a heavy Higgs sector, the increase in the SUSYbreaking scale has dramatic effects. The reason is that the lightest stop particle becomes much heavier in this case, and therefore the Higgs-boson masses are far away from the stop threshold. The mass shift coming from the computation of the Higgs-boson pole masses is never larger than about $3 \%$ in this case. In fact, this result depends only slightly on the exact value of the heavy Higgs-boson masses considered in Fig. 5 .

It is important to stress that the shift in the pole masses tends to increase the mass difference between the two neutral heavy Higgs states. In the effective-potential approximation, this mass difference is controlled by $\left|\mu A_{t}\right|$ (see [37], for instance), becoming smaller for larger values of the Higgs-boson masses (see the solid lines in Figs. 团 and 5). For the reasons explained above, the difference in pole masses between the two heavier states may actually be larger than the effects on light states, something that is quite apparent in Fig. 1 . This property may have important implications for physics at the LHC and at the future high-energy electron-positron and muon colliders.

We conclude this section with a brief discussion of the charged Higgs-boson pole mass $\widehat{M}_{H^{+}}$. As in the case of the neutral Higgs bosons, the effective-potential charged

\footnotetext{
${ }^{\dagger}$ Since $\mu$ is relatively large, chargino/neutralino on-shell effects are, instead, rather suppressed in the CPX scenario.

$\ddagger$ For such large values of the charged Higgs-boson mass, the lightest neutral Higgs boson $H_{1}$ has SM-like properties, due to the decoupling of the supersymmetric particles and the heavy Higgs bosons.
} 
Higgs-boson mass $M_{H^{+}}$differs from its pole value $\widehat{M}_{H^{+}}$. Either $M_{H^{+}}$or $\widehat{M}_{H^{+}}$could be used equally well as an input for the evaluation of the neutral Higgs-boson mass spectrum. If the same values for $M_{H^{+}}$and $\widehat{M}_{H^{+}}$were considered as inputs, they would result in slightly different values of $\operatorname{Re} \bar{m}_{12}^{2}$ and so in modest changes in the neutral Higgs-boson masses. Conversely, the mass difference $M_{H^{+}}-\widehat{M}_{H^{+}}$can be derived by finding values of $M_{H^{+}}$and $\widehat{M}_{H^{+}}$that give the same predictions for the neutral Higgs-boson mass. In Fig. 6, we present numerical estimates for this mass difference $M_{H^{+}}-\widehat{M}_{H^{+}}$as a function of the charged Higgs-boson pole mass $\widehat{M}_{H^{+}}$, for CPX scenarios with $\tan \beta=5$, and $M_{\mathrm{SUSY}}=0.5$ and $1 \mathrm{TeV}$. In these estimates, we also select different phases for the CP-violating parameters $m_{\tilde{g}}$ and $A_{t}: \arg \left(A_{t}\right)=\arg \left(m_{\tilde{g}}\right)=0$ (solid), $\arg \left(A_{t}\right)=90^{\circ}$ and $\arg \left(m_{\tilde{g}}\right)=0$ (dashed), $\arg \left(A_{t}\right)=\arg \left(m_{\tilde{g}}\right)=90^{\circ}$ (dotted), and $\arg \left(A_{t}\right)=-90^{\circ}$ and $\arg \left(m_{\tilde{g}}\right)=90^{\circ}$ (dash-dotted). We observe that the shifts in the charged Higgs-boson mass are larger in the CPX scenario with $M_{\mathrm{SUSY}}=0.5 \mathrm{TeV}$ shown in Fig. 6(a) than in the CPX scenario with $M_{\mathrm{SUSY}}=1 \mathrm{TeV}$ shown in Fig. 6(b), due to the fact that stop-sbottom production threshold effects are more significant in the first scenario.

\section{Conclusions}

In this article we have computed the Higgs-boson pole masses within the minimal supersymmetric standard model with explicit radiative CP violation in the Higgs sector [31. One-loop self-energy effects included in the definition of the pole masses are computed diagrammatically, while higher-order effects are computed via a RG improvement similar to that used previously in the effective-potential approximation.

In general, the effect on the Higgs-boson masses depends on the value of the supersymmetric particle masses: the closer the Higgs-boson mass is to the production threshold for a pair of supersymmetric particles, the larger becomes the difference between the pole masses and that computed in the effective-potential approach. Therefore, the relative shifts between pole and running masses are constrained to be much smaller in a light Higgs sector, where the masses are below the production threshold for top squarks. Still, for the $H_{1}$-boson sector, the absolute mass-difference value $\left|M_{H_{1}}-\widehat{M}_{H_{1}}\right|$ can be as large as $3 \mathrm{GeV}$. There is also a significant asymmetry between positive and negative values of $\arg \left(A_{t}\right)$, when $\arg \left(m_{\tilde{g}}\right)$ is large, e.g. $\arg \left(m_{\tilde{g}}\right)= \pm 90^{\circ}$, which is mainly associated with the supersymmetric threshold corrections to the top-quark Yukawa coupling.

The effects of computing the pole masses versus the running masses in the heavier Higgs sector may, instead, be very significant, particularly when the $H_{2}$-boson mass is 
close to the stop production threshold. We find shifts in masses that can be as large as $10 \%$ of the physical Higgs-boson masses. In addition, these mass shifts tend to increase the mass splitting between the two neutral heavy Higgs bosons, and therefore may have important implications for Higgs physics at the LHC, or at future Higgs factories such as linear electron-positron and muon colliders.

\section{Acknowledgements}

The work of C.W. is supported in part by the US DOE, Division of High-Energy Physics, under the contract no.: W-31-109-ENG-38. 


\section{A Higgs-Boson Couplings to Squarks}

We present in this Appendix the couplings of the Higgs bosons to squarks, incorporating $\mathrm{CP}$ violation.

The task of computing the Higgs-boson self-energies simplifies considerably if the Higgs-boson couplings are expressed in terms of $2 \times 2$ matrices in the weak basis of lefthanded and right-handed squarks, $\tilde{q}_{L}$ and $\tilde{q}_{R}$. Before listing the Higgs-boson couplings, it is useful to recall some of the standard relations associated with the squark sector of the third generation. The stop and sbottom mass matrices may conveniently be written as

$$
\widetilde{\mathcal{M}}_{q}^{2}=\left(\begin{array}{cc}
\tilde{M}_{Q}^{2}+m_{q}^{2}+\cos 2 \beta M_{Z}^{2}\left(T_{z}^{q}-Q_{q} \sin ^{2} \theta_{w}\right) & m_{q}\left(A_{q}^{*}-\mu R_{q}\right) \\
m_{q}\left(A_{q}-\mu^{*} R_{q}\right) & \tilde{M}_{q}^{2}+m_{q}^{2}+\cos 2 \beta M_{Z}^{2} Q_{q} \sin ^{2} \theta_{w}
\end{array}\right),
$$

with $q=t, b, Q_{t}=2 / 3, Q_{b}=-1 / 3, T_{z}^{t}=-T_{z}^{b}=1 / 2, R_{b}=\tan \beta=v_{2} / v_{1}, R_{t}=\cot \beta$, $M_{Z}^{2}=\frac{1}{4}\left(g_{w}^{2}+g^{\prime 2}\right) v^{2}$ and $\sin ^{2} \theta_{w}=g^{\prime 2} /\left(g_{w}^{2}+g^{\prime 2}\right)$. In (A.1), $\tilde{M}_{Q}^{2}$ and $\tilde{M}_{q}^{2}$ are soft SUSYbreaking masses for the left-handed and right-handed third-generation squarks. As usual, $\widetilde{\mathcal{M}}_{q}^{2}$ may be diagonalized by a unitary transformation $U^{q}$. The unitary matrix $U^{q}$ relates the weak $\left(\tilde{q}_{L}, \tilde{q}_{R}\right)$ and mass eigenstates $\left(\tilde{q}_{1}, \tilde{q}_{2}\right)$ through:

$$
\left(\begin{array}{c}
\tilde{q}_{L} \\
\tilde{q}_{R}
\end{array}\right)=U^{q}\left(\begin{array}{c}
\tilde{q}_{1} \\
\tilde{q}_{2}
\end{array}\right) ; \quad U^{q}=\left(\begin{array}{cc}
1 & 0 \\
0 & e^{i \delta_{q}}
\end{array}\right)\left(\begin{array}{cc}
\cos \theta_{q} & \sin \theta_{q} \\
-\sin \theta_{q} & \cos \theta_{q}
\end{array}\right)
$$

where $\delta_{q}=\arg \left(A_{q}-R_{q} \mu^{*}\right)$ and the mixing angles $\theta_{q}$ can uniquely be determined by

$$
\begin{aligned}
\cos \theta_{q} & =\frac{m_{q}\left|A_{q}-R_{q} \mu^{*}\right|}{\sqrt{m_{q}^{2}\left|A_{q}-R_{q} \mu^{*}\right|^{2}+\left[\left(\widetilde{\mathcal{M}}_{q}^{2}\right)_{11}-m_{\tilde{q}_{1}}^{2}\right]^{2}}}, \\
\sin \theta_{q} & =\frac{\left|\left(\widetilde{\mathcal{M}}_{q}^{2}\right)_{11}-m_{\tilde{q}_{1}}^{2}\right|}{\sqrt{m_{q}^{2}\left|A_{q}-R_{q} \mu^{*}\right|^{2}+\left[\left(\widetilde{\mathcal{M}}_{q}^{2}\right)_{11}-m_{\tilde{q}_{1}}^{2}\right]^{2}}} .
\end{aligned}
$$

Furthermore, the mass eigenvalues of $\widetilde{\mathcal{M}}_{q}^{2}$ are easily found to be

$$
\begin{aligned}
m_{\tilde{q}_{1}\left(\tilde{q}_{2}\right)}^{2}= & \frac{1}{2}\left\{\tilde{M}_{Q}^{2}+\tilde{M}_{q}^{2}+2 m_{q}^{2}+T_{z}^{q} \cos 2 \beta M_{Z}^{2}\right. \\
& \left.+(-) \sqrt{\left[\tilde{M}_{Q}^{2}-\tilde{M}_{q}^{2}+\cos 2 \beta M_{Z}^{2}\left(T_{z}^{q}-2 Q_{q} \sin ^{2} \theta_{w}\right)\right]^{2}+4 m_{q}^{2}\left|A_{q}^{*}-\mu R_{q}\right|^{2}}\right\} \cdot(A)
\end{aligned}
$$

In the following, we list individually the Higgs-boson couplings to top and bottom squarks, employing a $2 \times 2$-dimensional matrix representation:

$$
\Gamma^{\phi_{1} \tilde{t}^{*} \tilde{t}}=\left(\begin{array}{cc}
-\frac{1}{4}\left(g_{w}^{2}-\frac{1}{3} g^{2}\right) v_{1} & \frac{1}{\sqrt{2}} h_{t}^{*} \mu \\
\frac{1}{\sqrt{2}} h_{t} \mu^{*} & -\frac{1}{3} g^{\prime 2} v_{1}
\end{array}\right)
$$




$$
\begin{aligned}
& \Gamma^{\phi_{2} \tilde{t}^{*} \tilde{t}}=\left(\begin{array}{cc}
-\left|h_{t}\right|^{2} v_{2}+\frac{1}{4}\left(g_{w}^{2}-\frac{1}{3} g^{\prime 2}\right) v_{2} & -\frac{1}{\sqrt{2}} h_{t}^{*} A_{t}^{*} \\
-\frac{1}{\sqrt{2}} h_{t} A_{t} & -\left|h_{t}\right|^{2} v_{2}+\frac{1}{3} g^{\prime 2} v_{2}
\end{array}\right), \\
& \Gamma^{\phi_{1} \tilde{b}^{\tilde{b}} \tilde{b}}=\left(\begin{array}{cc}
-\left|h_{b}\right|^{2} v_{1}+\frac{1}{4}\left(g_{w}^{2}+\frac{1}{3} g^{\prime 2}\right) v_{1} & -\frac{1}{\sqrt{2}} h_{b}^{*} A_{b}^{*} \\
-\frac{1}{\sqrt{2}} h_{b} A_{b} & -\left|h_{b}\right|^{2} v_{1}+\frac{1}{6} g^{\prime 2} v_{1}
\end{array}\right), \\
& \Gamma^{\phi_{2} \tilde{b}^{*} \tilde{b}}=\left(\begin{array}{cc}
-\frac{1}{4}\left(g_{w}^{2}+\frac{1}{3} g^{2}\right) v_{2} & \frac{1}{\sqrt{2}} h_{b}^{*} \mu \\
\frac{1}{\sqrt{2}} h_{b} \mu^{*} & -\frac{1}{6} g^{\prime 2} v_{2}
\end{array}\right), \\
& \Gamma^{a_{1} \tilde{t}^{*} \tilde{t}}=\frac{i}{\sqrt{2}}\left(\begin{array}{cc}
0 & -h_{t}^{*} \mu \\
h_{t} \mu^{*} & 0
\end{array}\right), \quad \Gamma^{a_{2} \tilde{t}^{*} \tilde{t}}=\frac{i}{\sqrt{2}}\left(\begin{array}{cc}
0 & h_{t}^{*} A_{t}^{*} \\
-h_{t} A_{t} & 0
\end{array}\right) \text {, } \\
& \Gamma^{a_{1} \tilde{b}^{*} \tilde{b}}=\frac{i}{\sqrt{2}}\left(\begin{array}{cc}
0 & -h_{b}^{*} A_{b}^{*} \\
h_{b} A_{b} & 0
\end{array}\right), \quad \Gamma^{a_{2} \tilde{b}^{*} \tilde{b}}=\frac{i}{\sqrt{2}}\left(\begin{array}{cc}
0 & h_{b}^{*} \mu \\
-h_{b} \mu^{*} & 0
\end{array}\right), \\
& \Gamma^{\phi_{1}^{+} \tilde{t}^{*} \tilde{b}}=\left(\Gamma^{\phi^{-} \tilde{b}^{*} \tilde{t}}\right)^{\dagger}=\left(\begin{array}{cc}
-\frac{1}{\sqrt{2}}\left(\left|h_{b}\right|^{2}-\frac{1}{2} g_{w}^{2}\right) v_{1} & -h_{b}^{*} A_{b}^{*} \\
-h_{t} \mu^{*} & -\frac{1}{\sqrt{2}} h_{t} h_{b}^{*} v_{2}
\end{array}\right), \\
& \Gamma^{\phi_{2}^{+} \tilde{t}^{*} \tilde{b}}=\left(\Gamma^{\phi_{2}^{-} \tilde{b}^{*} \tilde{t}}\right)^{\dagger}=\left(\begin{array}{cc}
\frac{1}{\sqrt{2}}\left(\left|h_{t}\right|^{2}-\frac{1}{2} g_{w}^{2}\right) v_{2} & h_{b}^{*} \mu \\
h_{t} A_{t} & \frac{1}{\sqrt{2}} h_{t} h_{b}^{*} v_{1}
\end{array}\right) .
\end{aligned}
$$

Without loss of generality, the tree-level Yukawa couplings $h_{t}$ and $h_{b}$ can be taken to be real and positive [12], i.e. the $t$ - and $b$-quark masses are given by $m_{t}=\frac{1}{\sqrt{2}} h_{t} v_{2}$ and $m_{b}=\frac{1}{\sqrt{2}} h_{b} v_{1}$ at the tree level. Then, it is not difficult to verify that

$$
\begin{aligned}
& \widetilde{\mathcal{M}}_{t}^{2}=\operatorname{diag}\left(\tilde{M}_{Q}^{2}, \tilde{M}_{t}^{2}\right)-v_{1} \Gamma^{\phi_{1} \tilde{t}^{*} \tilde{t}}-v_{2} \Gamma^{\phi_{2} \tilde{t}^{*} \tilde{t}}, \\
& \widetilde{\mathcal{M}}_{b}^{2}=\operatorname{diag}\left(\tilde{M}_{Q}^{2}, \tilde{M}_{b}^{2}\right)-v_{1} \Gamma^{\phi_{1} \tilde{b}^{*} \tilde{b}}-v_{2} \Gamma^{\phi_{2} \tilde{b}^{*} \tilde{b}} .
\end{aligned}
$$

As well as trilinear couplings, Higgs bosons also have quadrilinear couplings to squarks. These additional couplings are given by

$$
\begin{aligned}
\Gamma^{\phi_{1} \phi_{1} \tilde{t}^{*} \tilde{t}} & =\Gamma^{a_{1} a_{1} \tilde{t}^{*} \tilde{t}}=\left(\begin{array}{cc}
-\frac{1}{4}\left(g_{w}^{2}-\frac{1}{3} g^{\prime 2}\right) & 0 \\
0 & -\frac{1}{3} g^{\prime 2}
\end{array}\right), \\
\Gamma^{\phi_{2} \phi_{2} \tilde{t}^{*} \tilde{t}} & =\Gamma^{a_{2} a_{2} \tilde{t}^{*} \tilde{t}}=\left(\begin{array}{cc}
-\left|h_{t}\right|^{2}+\frac{1}{4}\left(g_{w}^{2}-\frac{1}{3} g^{\prime 2}\right) & 0 \\
0 & -\left|h_{t}\right|^{2}+\frac{1}{3} g^{\prime 2}
\end{array}\right), \\
\Gamma^{\phi_{1} \phi_{1} \tilde{b}^{\tilde{b}} \tilde{b}} & =\Gamma^{a_{1} a_{1} \tilde{b}^{\tilde{b}} \tilde{b}}=\left(\begin{array}{cc}
-\left|h_{b}\right|^{2}+\frac{1}{4}\left(g_{w}^{2}+\frac{1}{3} g^{\prime 2}\right) & 0 \\
0 & -\left|h_{b}\right|^{2}+\frac{1}{6} g^{\prime 2}
\end{array}\right), \\
\Gamma^{\phi_{2} \phi_{2} \tilde{b}^{*} \tilde{b}} & =\Gamma^{a_{2} a_{2} \tilde{b}^{*} \tilde{b}}=\left(\begin{array}{cc}
-\frac{1}{4}\left(g_{w}^{2}+\frac{1}{3} g^{\prime 2}\right) & 0 \\
0 & -\frac{1}{6} g^{\prime 2}
\end{array}\right), \\
\Gamma^{\phi_{1}^{+} \phi_{1}^{-} \tilde{\tilde{t}}^{*} \tilde{t}} & =\left(\begin{array}{cc}
-\left|h_{b}\right|^{2}+\frac{1}{4}\left(g_{w}^{2}+\frac{1}{3} g^{\prime 2}\right) & 0 \\
0 & -\frac{1}{3} g^{\prime 2}
\end{array}\right),
\end{aligned}
$$




$$
\begin{aligned}
\Gamma^{\phi_{2}^{+} \phi_{2}^{-} \tilde{t}^{*} \tilde{t}} & =\left(\begin{array}{cc}
-\frac{1}{4}\left(g_{w}^{2}+\frac{1}{3} g^{2}\right) & 0 \\
0 & -\left|h_{t}\right|^{2}+\frac{1}{3} g^{\prime 2}
\end{array}\right), \\
\Gamma^{\phi_{1}^{+} \phi_{1}^{-} \tilde{b}^{*} \tilde{b}} & =\left(\begin{array}{cc}
-\frac{1}{4}\left(g_{w}^{2}-\frac{1}{3} g^{\prime 2}\right) & 0 \\
0 & -\left|h_{b}\right|^{2}+\frac{1}{6} g^{\prime 2}
\end{array}\right), \\
\Gamma^{\phi_{2}^{+} \phi_{2}^{-} \tilde{b}^{*} \tilde{b}} & =\left(\begin{array}{cc}
-\left|h_{t}\right|^{2}+\frac{1}{4}\left(g_{w}^{2}-\frac{1}{3} g^{\prime 2}\right) & 0 \\
0 & -\frac{1}{6} g^{\prime 2}
\end{array}\right) .
\end{aligned}
$$

\section{B Higgs-Boson Self-Energies}

In this Appendix, we derive analytic expressions for the neutral and charged Higgs-boson self-energies. We first consider the contributions of the third-generation squarks $\tilde{q}=\tilde{t}, \tilde{b}$ to the CP-even Higgs-boson self-energy transitions $\phi_{j} \rightarrow \phi_{i}$. Then, the CP-even Higgs-boson self-energies shown in Fig. 1 may be computed by

$$
\begin{aligned}
& \Pi_{i j}^{S,(a)}(s)=N_{c} \sum_{\tilde{q}=\tilde{t}, \tilde{b}} \int \frac{d^{D} k}{(2 \pi)^{D} i} \operatorname{Tr}\left[i \Delta^{\tilde{q}}(k+p) i \Gamma^{\phi_{i} \tilde{q}^{*} \tilde{q}} i \Delta^{\tilde{q}}(k) i \Gamma^{\phi_{j} \tilde{q}^{*} \tilde{q}}\right], \\
& \Pi_{i j}^{S,(b)}(s)=\delta_{i j} N_{c} \sum_{\tilde{q}=\tilde{t}, \tilde{b}} \int \frac{d^{D} k}{(2 \pi)^{D} i} \operatorname{Tr}\left[i \Delta^{\tilde{q}}(k) i \Gamma^{\phi_{i} \phi_{i} \tilde{q}^{*} \tilde{q}}\right],
\end{aligned}
$$

where $D=4-2 \varepsilon, N_{c}=3$ is the colour factor, and

$$
\Delta^{\tilde{q}}(k)=U^{q} \operatorname{diag}\left[\left(k^{2}-m_{\tilde{q}_{1}}^{2}\right)^{-1},\left(k^{2}-m_{\tilde{q}_{2}}^{2}\right)^{-1}\right] U^{q \dagger}
$$

is the squark-propagator matrix written in the weak basis. The unitary mixing matrix $U^{q}$ that relates the weak and mass eigenstates as well as the $2 \times 2$ coupling matrices $\Gamma^{\phi_{i} \tilde{q}^{*} \tilde{q}}$ and $\Gamma^{\phi_{i} \phi_{i} \tilde{q}^{*} \tilde{q}}$ were already presented in Appendix A.

To evaluate the self-energies in (B.1), it is more convenient to decompose the squark propagator as

$$
\Delta^{\tilde{q}}(k)=\Delta_{+}^{\tilde{q}}(k) \mathbf{1}_{2}+\Delta_{-}^{\tilde{q}}(k) U^{q} \tau_{3} U^{q \dagger},
$$

with $\mathbf{1}_{2}=\operatorname{diag}(1,1), \tau_{3}=\operatorname{diag}(1,-1)$ and

$$
\Delta_{ \pm}^{\tilde{q}}(k)=\frac{1}{2}\left(\frac{1}{k^{2}-m_{\tilde{q}_{1}}^{2}} \pm \frac{1}{k^{2}-m_{\tilde{q}_{2}}^{2}}\right) .
$$

With the above decomposition of the squark propagators, the neutral Higgs-boson selfenergies are analytically determined by

$$
\Pi_{i j}^{S,(a)}(s)=N_{c} \frac{1}{64 \pi^{2}} \sum_{\tilde{q}=\tilde{t}, \tilde{b}}\left\{\left[B_{0}\left(s, m_{\tilde{q}_{1}}^{2}, m_{\tilde{q}_{1}}^{2}\right)+B_{0}\left(s, m_{\tilde{q}_{2}}^{2}, m_{\tilde{q}_{2}}^{2}\right)\right.\right.
$$




$$
\begin{aligned}
& \left.+2 B_{0}\left(s, m_{\tilde{q}_{1}}^{2}, m_{\tilde{q}_{2}}^{2}\right)\right] \operatorname{Tr}\left(\Gamma^{\phi_{i} \tilde{q}^{*} \tilde{q}} \Gamma^{\phi_{j} \tilde{q}^{*} \tilde{q}}\right) \\
& +\left[B_{0}\left(s, m_{\tilde{q}_{1}}^{2}, m_{\tilde{q}_{1}}^{2}\right)-B_{0}\left(s, m_{\tilde{q}_{2}}^{2}, m_{\tilde{q}_{2}}^{2}\right)\right]\left[\operatorname{Tr}\left(\Gamma^{\phi_{i} \tilde{q}^{*} \tilde{q}} U^{q} \tau_{3} U^{q \dagger} \Gamma^{\phi_{j} \tilde{q}^{*} \tilde{q}}\right)\right. \\
& \left.+\operatorname{Tr}\left(\Gamma^{\phi_{i} \tilde{q}^{*} \tilde{q}} \Gamma^{\phi_{j} \tilde{q}^{*} \tilde{q}} U^{q} \tau_{3} U^{q \dagger}\right)\right]+\left[B_{0}\left(s, m_{\tilde{q}_{1}}^{2}, m_{\tilde{q}_{1}}^{2}\right)+B_{0}\left(s, m_{\tilde{q}_{2}}^{2}, m_{\tilde{q}_{2}}^{2}\right)\right. \\
& \left.\left.-2 B_{0}\left(s, m_{\tilde{q}_{1}}^{2}, m_{\tilde{q}_{2}}^{2}\right)\right] \operatorname{Tr}\left(\Gamma^{\phi_{i} \tilde{q}^{*} \tilde{q}} U^{q} \tau_{3} U^{q \dagger} \Gamma^{\phi_{j} \tilde{q}^{*} \tilde{q}} U^{q} \tau_{3} U^{q \dagger}\right)\right\} \\
\Pi_{i j}^{S,(b)}(s)= & -\delta_{i j} N_{c} \frac{1}{32 \pi^{2}} \sum_{\tilde{q}=\tilde{t}, \tilde{b}}\left\{\left[A_{0}\left(m_{\tilde{q}_{1}}^{2}\right)+A_{0}\left(m_{\tilde{q}_{2}}^{2}\right)\right] \operatorname{Tr}\left(\Gamma^{\phi_{i} \phi_{i} \tilde{q}^{*} \tilde{q}}\right)\right. \\
& \left.+\left[A_{0}\left(m_{\tilde{q}_{1}}^{2}\right)-A_{0}\left(m_{\tilde{q}_{2}}^{2}\right)\right] \operatorname{Tr}\left(\Gamma^{\phi_{i} \phi_{i} \tilde{q}^{*} \tilde{q}} U^{q} \tau_{3} U^{q \dagger}\right)\right\}
\end{aligned}
$$

Here, $B_{0}\left(s, m_{1}^{2}, m_{2}^{2}\right)$ and $A_{0}\left(m^{2}\right)$ are the usual Pasarino-Veltman one-loop functions [38]:

$$
\begin{aligned}
B_{0}\left(s, m_{1}^{2}, m_{2}^{2}\right)= & C_{\mathrm{UV}}-\ln \left(\frac{m_{1} m_{2}}{\mu^{2}}\right)+2+\frac{1}{s}\left[\left(m_{2}^{2}-m_{1}^{2}\right) \ln \left(\frac{m_{1}}{m_{2}}\right)\right. \\
& \left.+\lambda^{1 / 2}\left(s, m_{1}^{2}, m_{2}^{2}\right) \cosh ^{-1}\left(\frac{m_{1}^{2}+m_{2}^{2}-s}{2 m_{1} m_{2}}\right)\right], \\
A_{0}\left(m^{2}\right)= & m^{2}\left[1+B_{0}\left(0, m^{2}, m^{2}\right)\right]
\end{aligned}
$$

with $C_{\mathrm{UV}}=\frac{1}{\varepsilon}-\gamma_{E}+\ln 4 \pi$ and $\lambda(x, y, z)=(x-y-z)^{2}-4 y z$. In particular, $B_{0}\left(s, m_{1}^{2}, m_{2}^{2}\right)$ simplifies further when evaluated at $s=0$, to

$$
B_{0}\left(0, m_{1}^{2}, m_{2}^{2}\right)=C_{\mathrm{UV}}-\ln \left(\frac{m_{1} m_{2}}{\mu^{2}}\right)+1+\frac{m_{1}^{2}+m_{2}^{2}}{m_{1}^{2}-m_{2}^{2}} \ln \left(\frac{m_{2}}{m_{1}}\right) .
$$

Moreover, $U^{q} \tau_{3} U^{q \dagger}$ may be expressed entirely in terms of the elements of the squark mass matrix $\widetilde{\mathcal{M}}_{q}^{2}$ and of its squared mass-eigenvalues $m_{\tilde{q}_{1,2}}^{2}$, as

$$
U^{q} \tau_{3} U^{q \dagger}=\frac{1}{m_{\tilde{q}_{1}}^{2}-m_{\tilde{q}_{2}}^{2}}\left(\begin{array}{cc}
\left(\widetilde{\mathcal{M}}_{q}^{2}\right)_{11}-\left(\widetilde{\mathcal{M}}_{q}^{2}\right)_{22} & 2\left(\widetilde{\mathcal{M}}_{q}^{2}\right)_{12} \\
2\left(\widetilde{\mathcal{M}}_{q}^{2 *}\right)_{12} & \left(\widetilde{\mathcal{M}}_{q}^{2}\right)_{22}-\left(\widetilde{\mathcal{M}}_{q}^{2}\right)_{11}
\end{array}\right)
$$

The analytic expressions of the CP-odd Higgs-boson self-energies $\Pi_{i j}^{P}(s)$ may easily be recovered from the CP-even ones by replacing the coupling matrices $\Gamma^{\phi_{i} \tilde{q}^{*} \tilde{q}}$ and $\Gamma^{\phi_{i} \phi_{i} \tilde{q}^{*} \tilde{q}}$ with $\Gamma^{a_{i} \tilde{q}^{*} \tilde{q}}$ and $\Gamma^{a_{i} a_{i} \tilde{q}^{*} \tilde{q}}$ in $(\mathrm{B} .5)$ and $(\mathrm{B} .6)$.

The CP-violating scalar-pseudoscalar transitions $\phi_{j} \rightarrow a_{i}$ may also be calculated in a very analogous manner. Specifically, we find

$$
\begin{aligned}
\Pi_{i j}^{S P,(a)}(s)= & N_{c} \frac{1}{64 \pi^{2}} \sum_{\tilde{q}=\tilde{t}, \tilde{b}}\left\{\left[B_{0}\left(s, m_{\tilde{q}_{1}}^{2}, m_{\tilde{q}_{1}}^{2}\right)+B_{0}\left(s, m_{\tilde{q}_{2}}^{2}, m_{\tilde{q}_{2}}^{2}\right)\right.\right. \\
& \left.+2 B_{0}\left(s, m_{\tilde{q}_{1}}^{2}, m_{\tilde{q}_{2}}^{2}\right)\right] \operatorname{Tr}\left(\Gamma^{a_{i} \tilde{q}^{*} \tilde{q}} \Gamma^{\phi_{j} \tilde{q}^{*} \tilde{q}}\right) \\
& +\left[B_{0}\left(s, m_{\tilde{q}_{1}}^{2}, m_{\tilde{q}_{1}}^{2}\right)-B_{0}\left(s, m_{\tilde{q}_{2}}^{2}, m_{\tilde{q}_{2}}^{2}\right)\right]\left[\operatorname{Tr}\left(\Gamma^{a_{i} \tilde{q}^{*} \tilde{q}} U^{q} \tau_{3} U^{q \dagger} \Gamma^{\phi_{j} \tilde{q}^{*} \tilde{q}}\right)\right.
\end{aligned}
$$




$$
\begin{aligned}
& \left.+\operatorname{Tr}\left(\Gamma^{a_{i} \tilde{q}^{*} \tilde{q}} \Gamma^{\phi_{j} \tilde{q}^{*} \tilde{q}} U^{q} \tau_{3} U^{q^{\dagger}}\right)\right]+\left[B_{0}\left(s, m_{\tilde{q}_{1}}^{2}, m_{\tilde{q}_{1}}^{2}\right)+B_{0}\left(s, m_{\tilde{q}_{2}}^{2}, m_{\tilde{q}_{2}}^{2}\right)\right. \\
& \left.\left.-2 B_{0}\left(s, m_{\tilde{q}_{1}}^{2}, m_{\tilde{q}_{2}}^{2}\right)\right] \operatorname{Tr}\left(\Gamma^{a_{i} \tilde{q}^{*} \tilde{q}} U^{q} \tau_{3} U^{q^{\dagger}} \Gamma^{\phi_{j} \tilde{q}^{*} \tilde{q}} U^{q} \tau_{3} U^{q^{\dagger}}\right)\right\} .
\end{aligned}
$$

We have checked that $(\mathbb{B . 1 1})$ is in agreement with the original derivation presented in [8].

Finally, the analytic expressions of the charged Higgs-boson self-energy transitions $\phi_{j}^{-} \rightarrow \phi_{i}^{-}$take on an analogous form

$$
\begin{aligned}
\Pi_{i j}^{ \pm,(a)}(s)= & N_{c} \frac{1}{64 \pi^{2}}\left\{\left[B_{0}\left(s, m_{\tilde{t}_{1}}^{2}, m_{\tilde{b}_{1}}^{2}\right)+B_{0}\left(s, m_{\tilde{t}_{1}}^{2}, m_{\tilde{b}_{2}}^{2}\right)+B_{0}\left(s, m_{\tilde{t}_{2}}^{2}, m_{\tilde{b}_{1}}^{2}\right)\right.\right. \\
& \left.+B_{0}\left(s, m_{\tilde{t}_{2}}^{2}, m_{\tilde{b}_{2}}^{2}\right)\right] \operatorname{Tr}\left(\Gamma^{\phi_{i}^{+} \tilde{t}^{*} \tilde{b}} \Gamma^{\phi_{j}^{-} \tilde{b}^{*} \tilde{t}}\right) \\
& +\left[B_{0}\left(s, m_{\tilde{t}_{1}}^{2}, m_{\tilde{b}_{1}}^{2}\right)-B_{0}\left(s, m_{\tilde{t}_{1}}^{2}, m_{\tilde{b}_{2}}^{2}\right)+B_{0}\left(s, m_{\tilde{t}_{2}}^{2}, m_{\tilde{b}_{1}}^{2}\right)-B_{0}\left(s, m_{\tilde{t}_{2}}^{2}, m_{\tilde{b}_{2}}^{2}\right)\right] \\
& \times \operatorname{Tr}\left(\Gamma^{\phi_{i}^{+} \tilde{t}^{*} \tilde{b}} U^{b} \tau_{3} U^{b \dagger} \Gamma^{\phi_{j}^{-} \tilde{b}^{*} \tilde{t}}\right) \\
& +\left[B_{0}\left(s, m_{\tilde{t}_{1}}^{2}, m_{\tilde{b}_{1}}^{2}\right)+B_{0}\left(s, m_{\tilde{t}_{1}}^{2}, m_{\tilde{b}_{2}}^{2}\right)-B_{0}\left(s, m_{\tilde{t}_{2}}^{2}, m_{\tilde{b}_{1}}^{2}\right)-B_{0}\left(s, m_{\tilde{t}_{2}}^{2}, m_{\tilde{b}_{2}}^{2}\right)\right] \\
& \times \operatorname{Tr}\left(\Gamma^{\phi_{i}^{+} \tilde{t}^{*} \tilde{b}} \Gamma^{\phi_{j}^{-} \tilde{b}^{*} \tilde{t}} U^{t} \tau_{3} U^{t \dagger}\right) \\
& +\left[B_{0}\left(s, m_{\tilde{t}_{1}}^{2}, m_{\tilde{b}_{1}}^{2}\right)-B_{0}\left(s, m_{\tilde{t}_{1}}^{2}, m_{\tilde{b}_{2}}^{2}\right)-B_{0}\left(s, m_{\tilde{t}_{2}}^{2}, m_{\tilde{b}_{1}}^{2}\right)+B_{0}\left(s, m_{\tilde{t}_{2}}^{2}, m_{\tilde{b}_{2}}^{2}\right)\right] \\
& \left.\times \operatorname{Tr}\left(\Gamma^{\phi_{i}^{+} \tilde{t}^{*} \tilde{b}} U^{b} \tau_{3} U^{b \dagger} \Gamma^{\phi_{j}^{-} \tilde{b}^{*} \tilde{t}} U^{t} \tau_{3} U^{t \dagger}\right)\right\}, \\
\Pi_{i j}^{ \pm,(b)}(s)= & -\delta_{i j} N_{c} \frac{1}{32 \pi^{2}} \sum_{\tilde{q}=\tilde{t}, \tilde{b}}\left\{\left[A_{0}\left(m_{\tilde{q}_{1}}^{2}\right)+A_{0}\left(m_{\tilde{q}_{2}}^{2}\right)\right] \operatorname{Tr}\left(\Gamma_{i}^{\phi_{i}^{+} \phi_{i}^{-} \tilde{q}^{*} \tilde{q}}\right)\right. \\
& \left.+\left[A_{0}\left(m_{\tilde{q}_{1}}^{2}\right)-A_{0}\left(m_{\tilde{q}_{2}}^{2}\right)\right] \operatorname{Tr}\left(\Gamma^{\phi_{i}^{+} \phi_{i}^{-} \tilde{q}^{*} \tilde{q}} U^{q} \tau_{3} U^{q \dagger}\right)\right\} .
\end{aligned}
$$

In addition to squark loops, top and bottom quarks also contribute to the neutral and charged Higgs-boson self-energies. The $t$ - and $b$-quark contributions to the CP-even and CP-odd Higgs-boson self-energies are given explicitly by

$$
\begin{aligned}
& \Pi_{11}^{S,(c)}(s)=-N_{c} \frac{\left|h_{b}\right|^{2}}{8 \pi^{2}}\left[A_{0}\left(m_{b}^{2}\right)-\frac{1}{2}\left(s-4 m_{b}^{2}\right) B_{0}\left(s, m_{b}^{2}, m_{b}^{2}\right)\right], \\
& \Pi_{22}^{S,(c)}(s)=-N_{c} \frac{\left|h_{t}\right|^{2}}{8 \pi^{2}}\left[A_{0}\left(m_{t}^{2}\right)-\frac{1}{2}\left(s-4 m_{t}^{2}\right) B_{0}\left(s, m_{t}^{2}, m_{t}^{2}\right)\right], \\
& \Pi_{11}^{P,(c)}(s)=N_{c} \frac{\left|h_{b}\right|^{2}}{8 \pi^{2}}\left[A_{0}\left(m_{b}^{2}\right)-\frac{s}{2} B_{0}\left(s, m_{b}^{2}, m_{b}^{2}\right)\right], \\
& \Pi_{22}^{P,(c)}(s)=N_{c} \frac{\left|h_{t}\right|^{2}}{8 \pi^{2}}\left[A_{0}\left(m_{t}^{2}\right)-\frac{s}{2} B_{0}\left(s, m_{t}^{2}, m_{t}^{2}\right)\right],
\end{aligned}
$$

and we note that $\Pi_{12}^{S,(c)}(s)=\Pi_{12}^{P,(c)}(s)=0$, and also $\Pi_{i j}^{S P,(c)}(s)=0$, for $i, j=1,2$.

By analogy, the charged Higgs-boson self-energies also receive quantum corrections due to $t$ and $b$ quarks, viz.

$$
\Pi_{11}^{ \pm,(c)}(s)=-N_{c} \frac{\left|h_{b}\right|^{2}}{16 \pi^{2}}\left[A_{0}\left(m_{t}^{2}\right)+A_{0}\left(m_{b}^{2}\right)-\left(s-m_{t}^{2}-m_{b}^{2}\right) B_{0}\left(s, m_{t}^{2}, m_{b}^{2}\right)\right],
$$




$$
\begin{aligned}
& \Pi_{12}^{ \pm,(c)}(s)=N_{c} \frac{\left|h_{t} h_{b}\right|}{8 \pi^{2}} m_{t} m_{b} B_{0}\left(s, m_{b}^{2}, m_{t}^{2}\right) \\
& \Pi_{22}^{ \pm,(c)}(s)=-N_{c} \frac{\left|h_{t}\right|^{2}}{16 \pi^{2}}\left[A_{0}\left(m_{t}^{2}\right)+A_{0}\left(m_{b}^{2}\right)-\left(s-m_{t}^{2}-m_{b}^{2}\right) B_{0}\left(s, m_{t}^{2}, m_{b}^{2}\right)\right] .
\end{aligned}
$$

The charged and neutral Higgs-boson self-energies derived here are not UV-safe quantities. As has been discussed in Section 2, they can be renormalized in the $\overline{\mathrm{MS}}$ scheme by neglecting terms proportional to $C_{\mathrm{Uv}}$. However, even in the $\overline{\mathrm{MS}}$ scheme, one still has to take into consideration the relevant Higgs-boson tadpole CTs. Therefore, we quote the analytic results of the tadpole parameters $T_{\phi_{1,2}}$ and $T_{a_{1,2}}$. After taking into account the third-generation squark/quark loop effects displayed in Fig. 1(d) and (e), we obtain

$$
\begin{aligned}
T_{\phi_{i}}^{(d)}= & N_{c} \frac{1}{32 \pi^{2}} \sum_{\tilde{q}=\tilde{t}, \tilde{b}}\left\{\left[A_{0}\left(m_{\tilde{q}_{1}}^{2}\right)+A_{0}\left(m_{\tilde{q}_{2}}^{2}\right)\right] \operatorname{Tr}\left(\Gamma^{\phi_{i} \tilde{q}^{*} \tilde{q}}\right)\right. \\
& \left.+\left[A_{0}\left(m_{\tilde{q}_{1}}^{2}\right)-A_{0}\left(m_{\tilde{q}_{2}}^{2}\right)\right] \operatorname{Tr}\left(\Gamma^{\phi_{i} \tilde{q}^{*} \tilde{q}} U^{q} \tau_{3} U^{q \dagger}\right)\right\} \\
T_{\phi_{1}}^{(e)}= & N_{c} \frac{v_{1}\left|h_{b}\right|^{2}}{8 \pi^{2}} A_{0}\left(m_{b}^{2}\right), \\
T_{\phi_{2}}^{(e)}= & N_{c} \frac{v_{2}\left|h_{t}\right|^{2}}{8 \pi^{2}} A_{0}\left(m_{t}^{2}\right), \\
T_{a_{1}}^{(d)}= & N_{c} \frac{1}{32 \pi^{2}} \sum_{\tilde{q}=\tilde{t}, \tilde{b}}\left[A_{0}\left(m_{\tilde{q}_{1}}^{2}\right)-A_{0}\left(m_{\tilde{q}_{2}}^{2}\right)\right] \operatorname{Tr}\left(\Gamma^{a_{1} \tilde{q}^{*} \tilde{q}} U^{q} \tau_{3} U^{q \dagger}\right), \\
T_{a_{2}}^{(d)}= & -\tan \beta T_{a_{1}}^{(d)},
\end{aligned}
$$

with $T_{\phi_{1,2}}=T_{\phi_{1,2}}^{(d)}+T_{\phi_{1,2}}^{(e)}$ and $T_{a_{1,2}}=T_{a_{1,2}}^{(d)}$. 


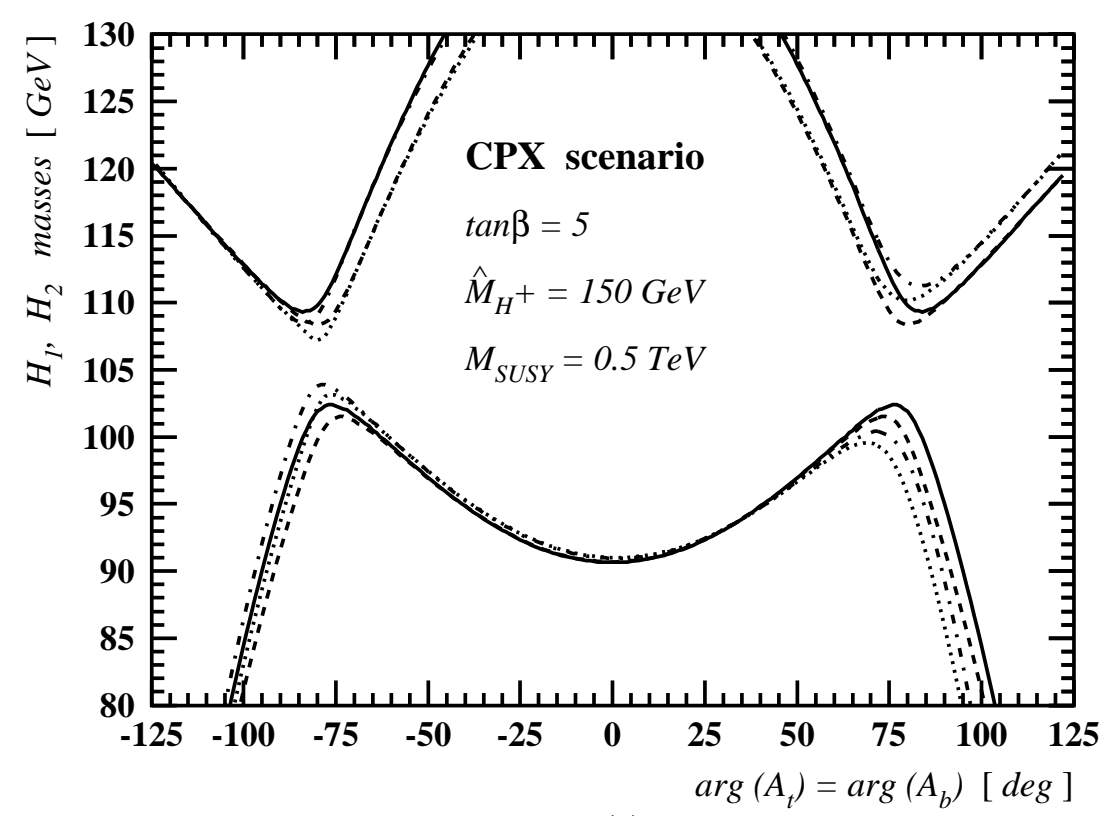

(a)

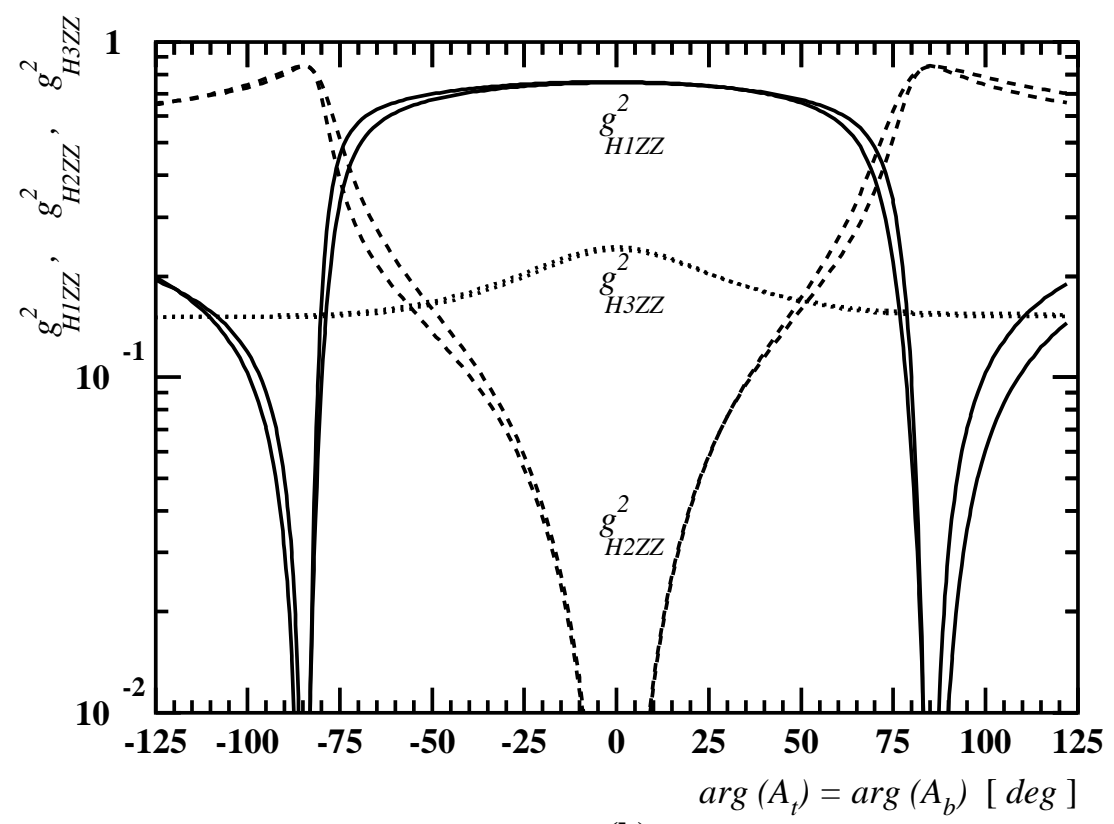

(b)

Figure 2: Numerical estimates of (a) the $H_{1,2^{-}}$effective-potential and pole masses and (b) $g_{H_{i} Z Z}^{2}$ as functions of $\arg \left(A_{t}\right)$, in a CPX scenario with $M_{\mathrm{SUSY}}=0.5 \mathrm{TeV}, \arg \left(m_{\tilde{g}}\right)=0$ and $90^{\circ}$. In plot (a), the effective-potential mass $M_{H_{1}}\left(M_{H_{2}}\right)$ is indicated by a solid (dashdotted) line for $\arg \left(m_{\tilde{g}}\right)=0\left(90^{\circ}\right)$, and its pole mass $\widehat{M}_{H_{1}}\left(\widehat{M}_{H_{2}}\right)$ by a dashed (dotted) line for $\arg \left(m_{\tilde{g}}\right)=0\left(90^{\circ}\right)$. 


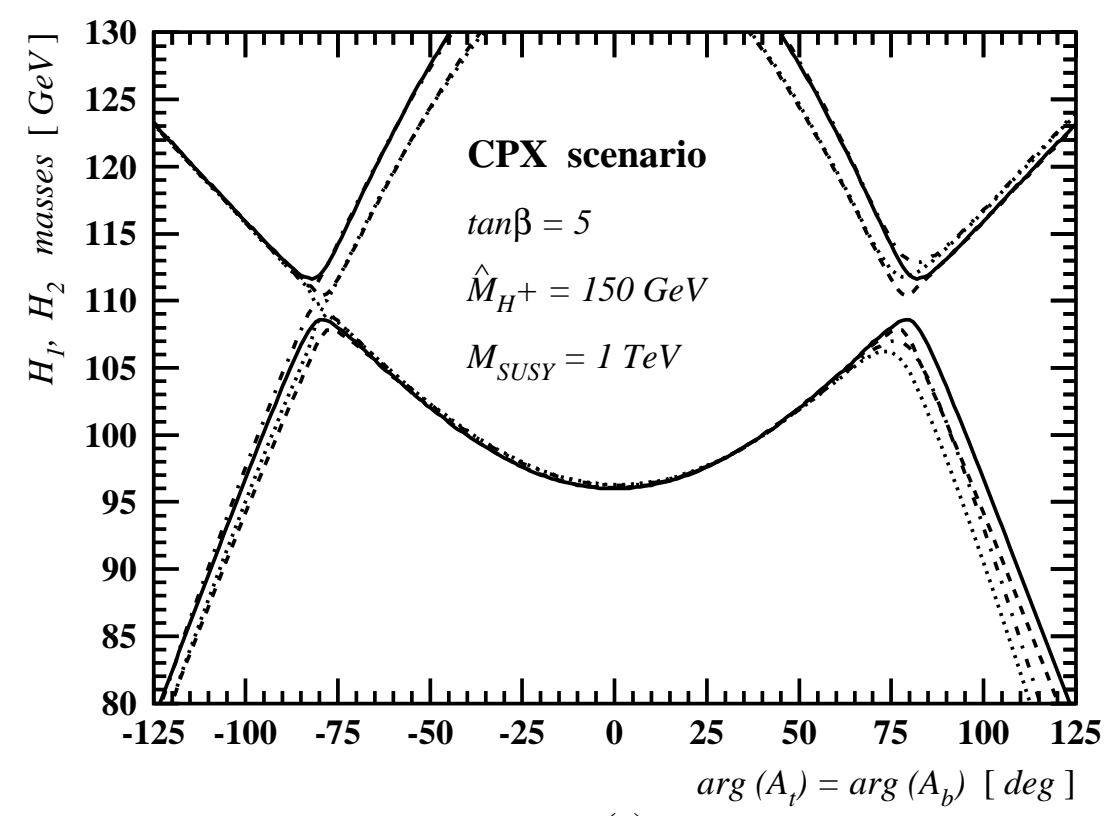

(a)

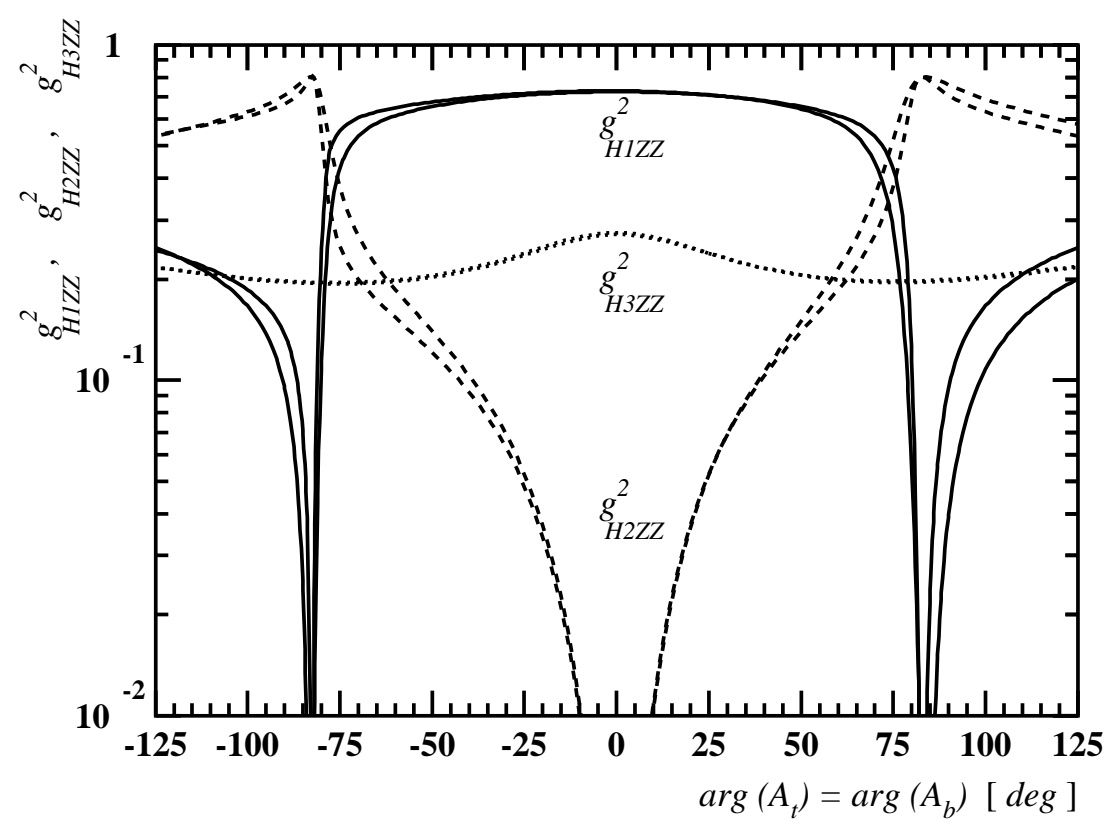

(b)

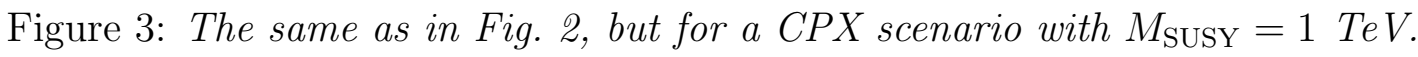



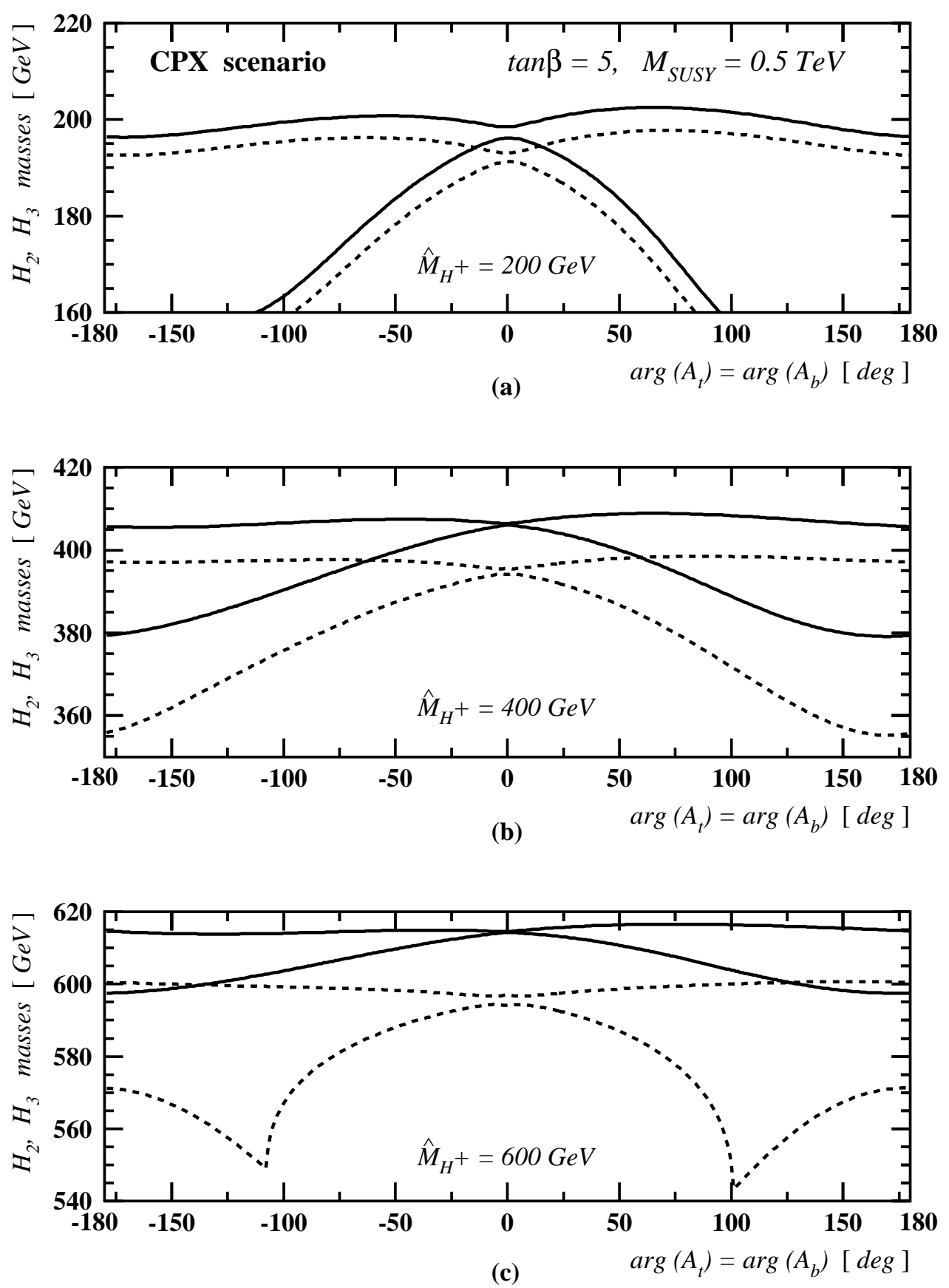

Figure 4: Numerical estimates of the two heaviest $H_{2}$-and $H_{3}$-boson masses versus $\arg \left(A_{t}\right)$ for different charged Higgs-boson pole masses in a CPX scenario with $\arg \left(m_{\tilde{g}}\right)=90^{\circ}$. Effective-potential masses are indicated by solid lines and pole masses by dashed ones. 

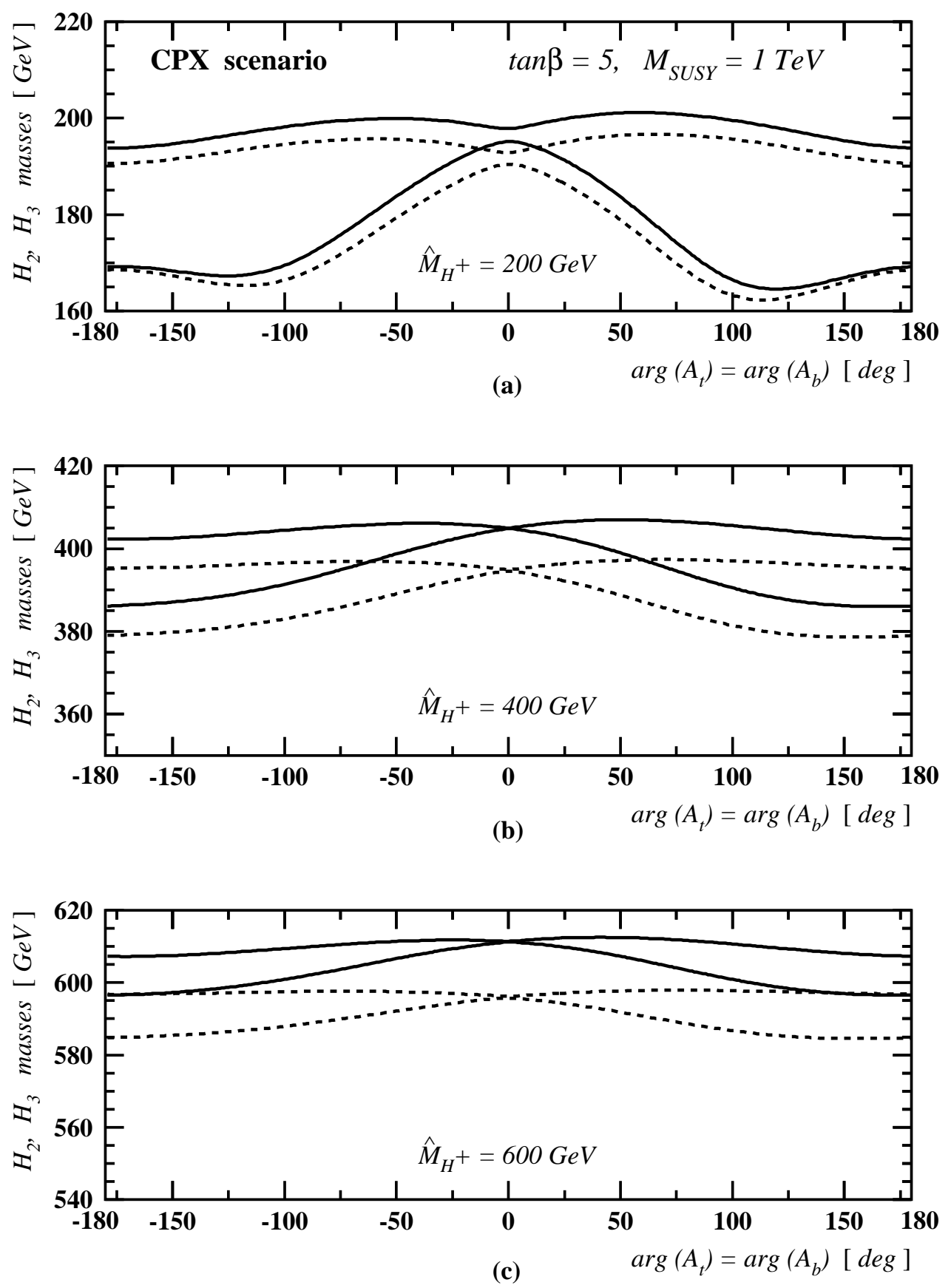

Figure 5: The same as in Fig. 团, but for a CPX scenario with $M_{\mathrm{SUSY}}=1 \mathrm{TeV}$. 

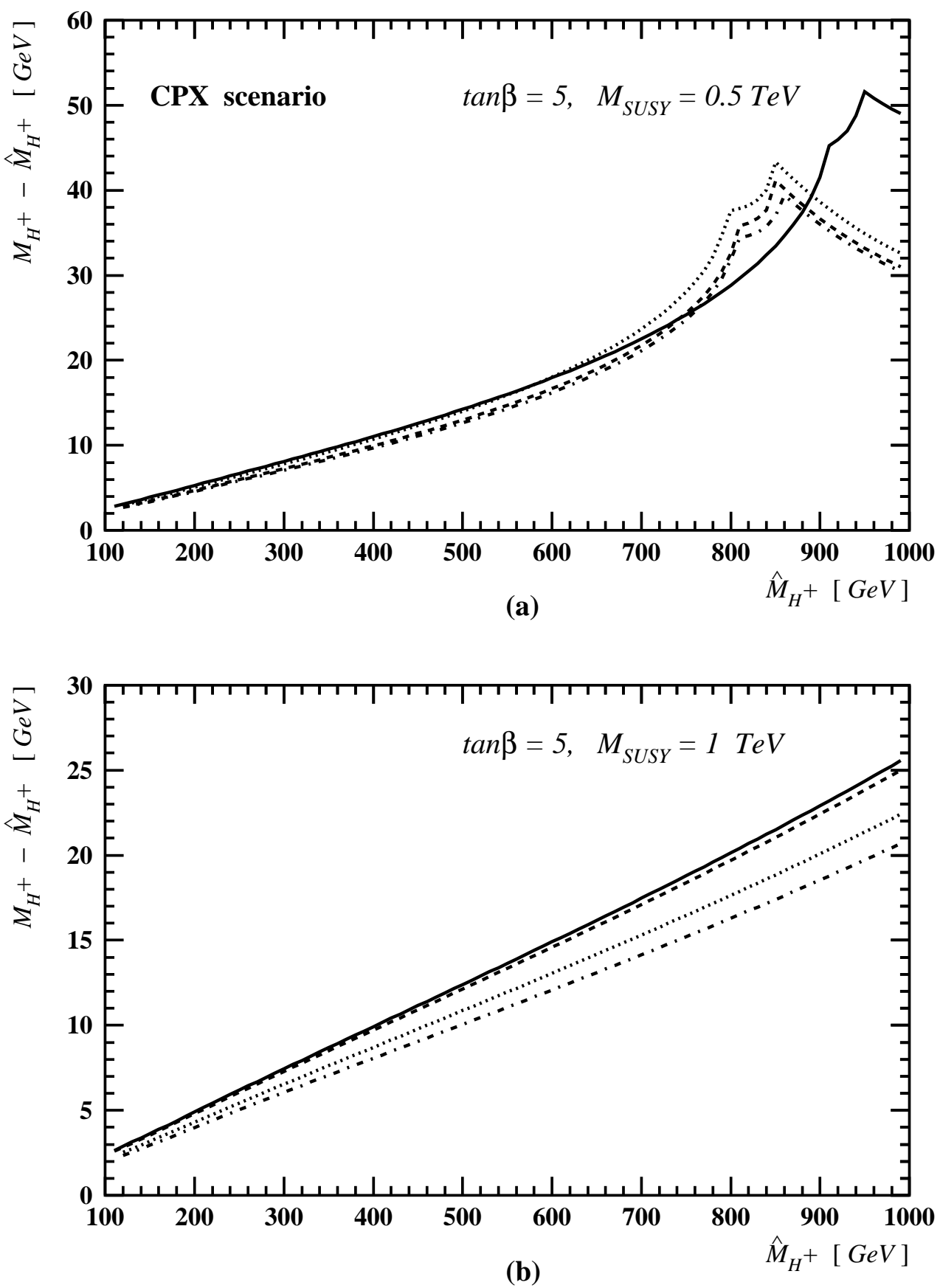

Figure 6: Numerical values of the difference between the effective-potential and the pole mass of the charged Higgs boson, $M_{H^{+}}-\widehat{M}_{H^{+}}$, as a function of $\widehat{M}_{H^{+}}$, for a CPX scenario with (a) $M_{\mathrm{SUSY}}=0.5 \mathrm{TeV}$ and (b) $M_{\mathrm{SUSY}}=1 \mathrm{TeV}$. The different types of lines correspond to $\arg \left(A_{t}\right)=\arg \left(m_{\tilde{g}}\right)=0$ (solid), $\arg \left(A_{t}\right)=90^{\circ}$ and $\arg \left(m_{\tilde{g}}\right)=0$ (dashed), $\arg \left(A_{t}\right)=$ $\arg \left(m_{\tilde{g}}\right)=90^{\circ}$ (dotted), and $\arg \left(A_{t}\right)=-90^{\circ}$ and $\arg \left(m_{\tilde{g}}\right)=90^{\circ}$ (dash-dotted). 


\section{References}

[1] For reviews, see, H.P. Nilles, Phys. Rep. 110 (1984) 1; H. Haber and G. Kane, Phys. Rep. 117 (1985) 75; J.F. Gunion, H.E. Haber, G.L. Kane and S. Dawson, The Higgs Hunter's Guide, (Addison-Wesley, Reading, MA, 1990).

[2] S. Dimopoulos and H. Georgi, Nucl. Phys. B193 (1981) 150; S. Dimopoulos, S. Raby and F. Wilczek, Phys. Rev. D24 (1981) 1681; M. Carena, S. Pokorski and C.E.M. Wagner, Nucl. Phys. B406 (1993) 59.

[3] V.A. Kuzmin, V.A. Rubakov and M.E. Shaposhnikov, Phys. Lett. B155 (1985) 36.

[4] For recent studies, see, M. Carena, M. Quirós and C.E.M. Wagner, Nucl. Phys. B524 (1998) 3; M. Laine and K. Rummukainen, Phys. Rev. Lett. 80 (1998) 5259 and Nucl. Phys. B535 (1998) 423; K. Funakubo, Prog. Theor. Phys. 101 (1999) 415 and 102 (1999) 389; J. Grant and M. Hindmarsh, Phys. Rev. D59 (1999) 116014; S. Davidson, M. Losada and A. Riotto, Phys. Rev. Lett. 84 (2000) 4284; A.B. Lahanas, V.C. Spanos and V. Zarikas, Phys. Lett. B472 (2000) 119; M. Carena, J.M. Moreno, M. Quiros, M. Seco and C.E.M. Wagner, Nucl. Phys. B599 (2001) 158; M. Laine and K. Rummukainen, Nucl. Phys. B597 (2001) 23; S.J. Huber, P. John and M.G. Schmidt, Eur. Phys. J. C20 (2001) 695.

[5] H. Goldberg, Phys. Rev. Lett. 50 (1983) 1419; J. Ellis, J.S. Hagelin, D.V. Nanopoulos, K.A. Olive and M. Srednicki, Nucl. Phys. B238 (1984) 453; M. Drees and M.M. Nojiri, Phys. Rev. D47 (1993) 376; M.E. Gomez, G. Lazarides and C. Pallis, Phys. Lett. B487 (2000) 313; P. Gondolo and K. Freese, hep-ph/990839; T. Falk, A. Ferstl and K.A. Olive, Astropart. Phys. 13 (2000) 301; S.Y. Choi, S.-C. Park, J.H. Jang and H.S. Song, Phys. Rev. D64 (2001) 015006. For a recent study, see J. R. Ellis, T. Falk, G. Ganis, K. A. Olive and M. Srednicki, Phys. Lett. B510 (2001) 236.

[6] J. Ellis, G. Ridolfi and F. Zwirner, Phys. Lett. B257 (1991) 83; M.S. Berger, Phys. Rev. D41 (1990) 225; Y. Okada, M. Yamaguchi and T. Yanagida, Prog. Theor. Phys. 85 (1991) 1; Phys. Lett. B262 (1991) 54; H.E. Haber and R. Hempfling, Phys. Rev. Lett. 66 (1991) 1815.

[7] ALEPH, DELPHI, L3 and OPAL Collaborations, LEP Electroweak Working Group, SLD Heavy Flavor Group and SLD Electroweak Group, CERN-EP-2001-021, hepex/0103048; see also http://lepewWg.web.cern.ch/LEPEWWG/Welcome.html. 
[8] A. Pilaftsis, Phys. Rev. D58 (1998) 096010 and Phys. Lett. B435 (1998) 88.

[9] A. Pilaftsis and C.E.M. Wagner, Nucl. Phys. B553 (1999) 3.

[10] D.A. Demir, Phys. Rev. D60 (1999) 055006.

[11] S.Y. Choi, M. Drees and J.S. Lee, Phys. Lett. B481 (2000) 57.

[12] M. Carena, J. Ellis, A. Pilaftsis and C.E.M. Wagner, Nucl. Phys. B586 (2000) 92.

[13] G.L. Kane and L.-T. Wang, Phys. Lett. B488 (2000) 383.

[14] S.Y. Choi and J.S. Lee, Phys. Rev. D61 (2000) 015003; S.Y. Choi, K. Hagiwara and J.S. Lee, Phys. Rev. D64 (2001) 032004 and hep-ph/0110138.

[15] T. Ibrahim and P. Nath, Phys. Rev. D63 (2001) 035009.

[16] M. Carena, J. Ellis, A. Pilaftsis and C.E.M. Wagner, Phys. Lett. B495 (2000) 155.

[17] T. Ibrahim, Phys. Rev. D64 (2001) 035009.

[18] S. Heinemeyer, Eur. Phys. J. C22 (2001) 521.

[19] J. Kodaira, Y. Yasui and K. Sasaki, Phys. Rev. D50 (1994) 7035.

[20] J.A. Casas, J.R. Espinosa, M. Quirós and A. Riotto, Nucl. Phys. B436 (1995) 3; (E) B439 (1995) 466.

[21] M. Carena, J.R. Espinosa, M. Quirós and C.E.M. Wagner, Phys. Lett. B355 (1995) 209.

[22] M. Carena, M. Quiros and C.E.M. Wagner, Nucl. Phys. B461 (1996) 407; H.E. Haber, R. Hempfling and A.H. Hoang, Z. Phys. C75 (1997) 539.

[23] H.E. Haber and R. Hempfling, Phys. Rev. D48 (1993) 4280.

[24] S. Heinemeyer, W. Hollik, G. Weiglein, Phys. Lett. B440 (1998) 296 and Eur. Phys. J. C9 (1999) 343; M. Carena, H.E. Haber, S. Heinemeyer, W. Hollik, C.E.M. Wagner and G. Weiglein, Nucl. Phys. B580 (2000) 29; J.R. Espinosa and R.J. Zhang, Nucl. Phys. B586 (2000) 3 and JHEP 0003 (2000) 026.

[25] R. Garisto and J.D. Wells, Phys. Rev. D55 (1997) 1611.

[26] J. R. Espinosa and I. Navarro, Nucl. Phys. B615 (2001) 82. 
[27] S. Heinemeyer, private communication.

[28] A. Pilaftsis, Phys. Rev. Lett. 77 (1996) 4996 and Nucl. Phys. B504 (1997) 61.

[29] J. Papavassiliou and A. Pilaftsis, Phys. Rev. Lett. 75 (1995) 3060; Phys. Rev. D53 (1996) 2128; Phys. Rev. D54 (1996) 5315; Phys. Rev. Lett. 80 (1998) 2785 and Phys. Rev. D58 (1998) 053002.

[30] A. Sirlin, Phys. Rev. Lett. 67 (1991) 2127 and Phys. Lett. B267 (1991) 240; R.G. Stuart, Phys. Lett. B262 (1991) 113 and Phys. Rev. Lett. 70 (1993) 3193; H. Veltman, Z. Phys. C62 (1994) 35.

[31] The Fortran code $\mathrm{cph}+\mathrm{f}$ used in this paper is available at http://home.cern.ch/p/pilaftsi/www/.

[32] D. Chang, W.-Y. Keung and A. Pilaftsis, Phys. Rev. Lett. 82 (1999) 900 and 83 (1999) 3972 (E); A. Pilaftsis, Phys. Lett. B471 (1999) 174; D. Chang, W.-F. Chang and W.-Y. Keung, Phys. Lett. B478 (2000) 239.

[33] A. Pilaftsis, Phys. Rev. D62 (2000) 016007.

[34] J. Dai, H. Dykstra, R.G. Leigh, S. Paban, and D.A. Dicus, Phys. Lett. B237 (1990) 216 and B242 (1990) 547 (E).

[35] For a recent updated analysis, see S. Abel, S. Khalil and O. Lebedev, Nucl. Phys. B606 (2001) 151.

[36] T. Ibrahim and P. Nath, Phys. Lett. B418 (1998) 98; Phys. Rev. D57 (1998) 478; D58 (1998) 019901 (E) and Phys. Rev. D58 (1998) 111301; M. Brhlik, G.J. Good and G.L. Kane, Phys. Rev. D59 (1999) 115004; M. Brhlik, L. Everett, G.L. Kane and J. Lykken, Phys. Rev. Lett. 83 (1999) 2124.

[37] M. Carena, S. Mrenna and C.E.M. Wagner, Phys. Rev. D60 (1999) 075010 and Phys. Rev. D62 (2000) 055008.

[38] G. Pasarino and M. Veltman, Nucl. Phys. B160 (1979) 151. 